\title{
Planes of Isotropic Poisson's Ratio in Anisotropic Crystalline Solids
}

\section{Shrikanth S. ${ }^{\mathrm{a}}$, Kevin M. Knowles ${ }^{\mathrm{b}}$, Suresh Neelakantan ${ }^{\mathrm{a}, *}$ and Rajesh Prasad ${ }^{\mathrm{a}}$}

${ }^{a}$ Department of Materials Science and Engineering, Indian Institute of Technology Delhi, Hauz Khas, New Delhi-110016, India

${ }^{b}$ Department of Materials Science and Metallurgy, University of Cambridge, 27 Charles Babbage Road, Cambridge CB3 OFS, UK.

*Corresponding author: TX-200N, Department of Materials Science and Engineering, Indian Institute of Technology Delhi, Hauz Khas, New Delhi - 110 016, India.

E-mail: sureshn@iitd.ac.in

\section{Acknowledgements}

This work is part of the Early Career Research (ECR) grant award (Ref. no.: ECR/2016/001953) funded by Science and Engineering Research Board (SERB) of Department of Science and Technology (DST), India. Dr. Suresh Neelakantan acknowledges the support.

Declaration of interest: none 


\begin{abstract}
It is shown that loading directions can exist in all single crystals for which Poisson's ratio is isotropic in the plane transverse to the loading direction. The body diagonals and the cube axes of cubic crystals, the four-fold axis in tetragonal crystals and the six-fold axis in hexagonal crystals are examples of such loading directions. However, these loading directions do not necessarily just lie only along high rotational symmetry axes. A new threedimensional "anisotropy surface" is introduced to represent the degree of anisotropy of the Poisson's ratio within the plane normal to a loading direction for a particular choice of single crystal.
\end{abstract}

Keywords: Anisotropy; Crystals; Elastic solids; Poisson's ratio

\title{
1. Introduction
}

Poisson's ratio $(v)$ is defined (Poisson, 1827) as the negative of the ratio of the strain along the transverse direction $\left(\varepsilon_{t}\right)$ to the strain along the loading direction $\left(\varepsilon_{l}\right)$ for a rod under uniaxial tension. Common isotropic materials such as glasses, polycrystalline metals and rubber narrow when they are stretched and expand laterally when they are compressed. Most isotropic materials used in engineering applications have values of Poisson's ratio close to 0.3 .

For single-crystalline materials, the Poisson's ratio can be highly anisotropic - positive, negative or zero - within the same material as a function of loading direction and transverse direction. The directional dependence of Poisson's ratio has been widely studied over the past four decades (see, for example, Turley and Sines, 1971, Gunton and Saunders, 1972, Li, 1976, Ballato, 1996, Baughman et al., 1998, Hayes and Shuvalov, 1998, Lubarda and Meyers, 1999, Norris, 2006a, Norris 2006b, Paszkiewicz and Wolski 2007a, Paszkiewicz and 
Wolski 2007b, Brańka et al., 2009, Lethbridge et al., 2010, Brańka et al., 2011, Adachi et al., 2018). It has also been established that the Poisson's ratios of anisotropic solids do not have any limits (Ting and Chen, 2005), unlike isotropic solids.

Negative Poisson's ratio or "auxetic" behaviour is well-known. Materials with negative Poisson's ratio were named "auxetic" materials or simply "auxetics" (from the Greek word

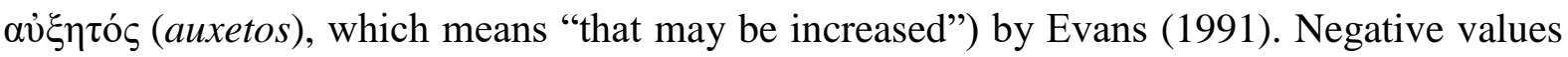
of Poisson's ratio were first reported by Love (1944) for pyrite. Naturally existing auxetic single crystals generally exhibit small negative values of Poisson ratio in the range $-1 \leq v \leq$ 0 , while artificial materials or structures can be designed to show high auxetic values in the range $-20 \leq v \leq 0$ (Neelakantan et al., 2015).

It is also known that there are certain high symmetry loading directions for which the Poisson's ratio is the same along all the transverse directions. A schematic of this is shown in Figure 1(a) in comparison with a more general loading situation in the schematic of Figure 1(b). Observed loading directions of such kind are the $\langle 111\rangle$ and $\langle 100\rangle$ axes in cubic materials (Baughman et al., 1998), the four-fold axis in tetragonal materials (Gunton and Saunders, 1975), and the six-fold axis in hexagonal materials (Li, 1976). Ballato (1996) has proved that when loaded along these symmetry directions, the Poisson's ratio is isotropic for all transverse directions. An example of a loading direction other than the axes of symmetry where the Poisson's ratio is isotropic in the transverse plane has been reported in the tetragonal metal indium (Gunton and Saunders, 1975), without its significance being explained explicitly.

Other than for axes of a high degree of rotational symmetry, it is evident that the existence of loading directions for which the Poisson's ratio is isotropic in the transverse plane has not been systematically investigated. The three-fold axes in arsenic, antimony and bismuth (trigonal) have also been reported to be such loading directions (Gunton and Saunders, 1972), but the present authors are unaware of a formal proof that the three-fold axes in trigonal materials are always loading directions for which Poisson's ratio is isotropic 
in the transverse plane. It is demonstrated in the current work that a three-fold, four-fold, or six-fold symmetry about the loading axis is a sufficient, but not a necessary, condition for Poisson's ratio to be isotropic in the plane transverse to the loading direction. Other loading directions without a high rotational symmetry in materials of all seven crystal systems are presented here for which Poisson's ratio is isotropic in the transverse plane, together with the mathematical formulae to determine the directions.

\section{Method}

\subsection{General Expression for Poisson's Ratio}

Our goal is to generate formulae defining the planes transverse to a particular loading direction for which Poisson's ratio is isotropic. For this, it is necessary to evaluate the Poisson's ratio along all transverse directions for an arbitrary loading direction in a given crystal in terms of its known Voigt compliances $s_{i j}$ (a $6 \times 6$ matrix with $\left.i, j=1-6\right)$. The measured compliances are referred to a standard orthonormal Cartesian coordinate system fixed with respect to the crystallographic axes (Nye, 1957). This set of axes is labelled $x_{1}, x_{2}, x_{3}$ (Figure 2) and will be referred to as the Voigt coordinate system here. A subscript $V$ has been used to specify all the directions referred to within this orthonormal coordinate system. An arbitrary loading direction, $\widehat{\mathbf{n}}=\left[n_{1}, n_{2}, n_{3}\right]_{V}$ and a transverse direction, $\widehat{\mathbf{m}}=$ $\left[m_{1}, m_{2}, m_{3}\right]_{V}$ have been chosen to determine the Poisson ratio $v_{m n}$. It is convenient to introduce another orthonormal coordinate system $x_{1}^{\prime}, x_{2}^{\prime}, x_{3}^{\prime}$ so that $x_{3}^{\prime}$ and $x_{1}^{\prime}$ are along $\hat{\mathbf{n}}$ and $\widehat{\mathbf{m}}$, respectively. This will be referred to as the Poisson coordinate system, where the Poisson's ratio has a simple form:

$$
v_{m n}=-\frac{\varepsilon_{m}}{\varepsilon_{n}}=-\frac{\varepsilon_{11}^{\prime}}{\varepsilon_{33}^{\prime}}=-\frac{s_{13}^{\prime}}{s_{33}^{\prime}} .
$$


However, as $s_{13}^{\prime}$ and $s_{33}^{\prime}$ are not measured directly, they have to be related to the measured values $s_{i j}$ in the Voigt coordinate system. This can be done through the tensor relations between the two coordinate systems:

$$
s_{13}^{\prime}=s_{1133}^{\prime}=a_{1 p} a_{1 q} a_{3 r} a_{3 s} s_{p q r s}
$$

and

$$
s_{33}^{\prime}=s_{3333}^{\prime}=a_{3 p} a_{3 q} a_{3 r} a_{3 s} s_{p q r s}
$$

Here, $s_{p q r s}$ are the tensor compliances and $a_{i j}$ are the elements of the matrix of direction cosines between the $x_{i}^{\prime}$ axis of the Poisson coordinate system and the $x_{j}$ axis of the Voigt coordinate system:

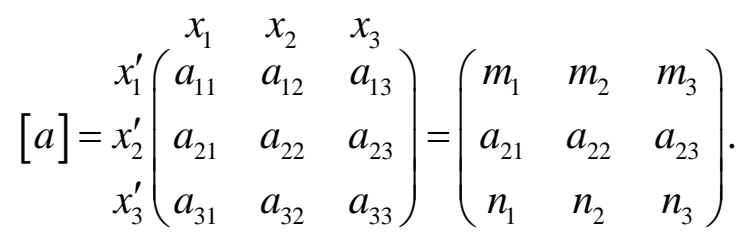

In equations (2) and (3), the Einstein summation convention is used for $p, q, r$ and $s$ taking all values from 1 to 3 .

As stated above, we need to examine all transverse directions $(\widehat{\mathbf{m}})$ for a given loading direction ( $(\hat{\mathbf{n}})$. Therefore, a new coordinate system has to be defined for each of these transverse directions so that $x_{3}^{\prime}$ lies along $\hat{\mathbf{n}}$ and $x_{1}^{\prime}$ lies along $\widehat{\mathbf{m}}$. Before we define the new coordinate system, $x_{1}^{\prime}, x_{2}^{\prime}, x_{3}^{\prime}$, it is convenient to define an intermediate right-handed orthonormal system $x_{1}^{\beta}, x_{2}^{\beta}, x_{3}^{\beta}$ so that $x_{1}^{\beta}$ and $x_{2}^{\beta}$ are two directions normal to $\hat{\mathbf{n}}$, and $x_{3}^{\beta}$ is parallel to $\hat{\mathbf{n}}$. The unit vector along the loading direction can be expressed in terms of its spherical coordinates $(r, \theta, \varphi)$ as

$$
\left[n_{1}, n_{2}, n_{3}\right]_{V}=[\cos \theta \sin \varphi, \sin \theta \sin \varphi, \cos \varphi]_{V}
$$


where $\theta$ is the azimuthal angle (the angle between the $x_{1}$ axis and the projection of $\hat{\mathbf{n}}$ on the $x_{1}, x_{2}$ plane measured in the anti-clockwise direction looking along $-x_{3}$ ) and $\varphi$ is the polar angle (the angle from the $x_{3}$ axis to $\hat{\mathbf{n}}$ ), as shown in Figure 2.

The $x_{1}^{\beta}$ axis can be chosen to be any direction in the transverse plane. However, it is convenient to choose a direction lying along the intersection of the transverse plane with the $\left(x_{1}, x_{2}\right)$ plane of the Voigt system. The positive sense of $x_{1}^{\beta}$ is arbitrarily chosen along $[-\sin \theta, \cos \theta, 0]_{V}$. Therefore, we have the matrix of direction cosines $a_{i j}^{\beta}$ as the following:

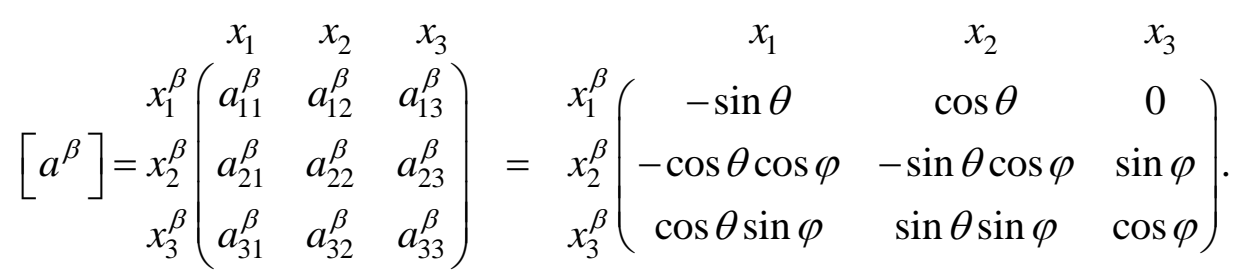

The required coordinate system $x_{1}^{\prime}, x_{2}^{\prime}, x_{3}^{\prime}$ can be obtained by an anti-clockwise rotation of the $x_{1}^{\beta}, x_{2}^{\beta}, x_{3}^{\beta}$ coordinate system about $x_{3}^{\beta}$ by an angle $\alpha$ varying from $0^{\circ}$ to $180^{\circ}$. Due to the centrosymmetry of strains, there is no need to vary $\alpha$ from $0^{\circ}$ to $360^{\circ}$. The matrix of direction cosines for the transformation from $x_{j}^{\beta}$ to $x_{i}^{\prime}$ is given by

$$
\begin{aligned}
& x_{1}^{\beta} \quad x_{2}^{\beta} \quad x_{3}^{\beta}
\end{aligned}
$$

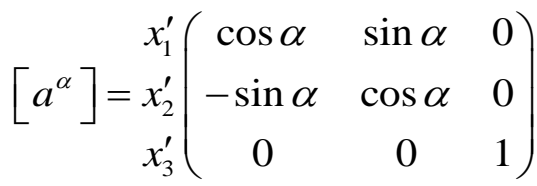

The matrix for transformation from the Voigt to the Poisson coordinates is given by

$$
[a]=\left[a^{\alpha}\right]\left[a^{\beta}\right]
$$

so that 


$$
\begin{aligned}
& \begin{array}{lll}
x_{1} & x_{2} & x_{3}
\end{array}
\end{aligned}
$$

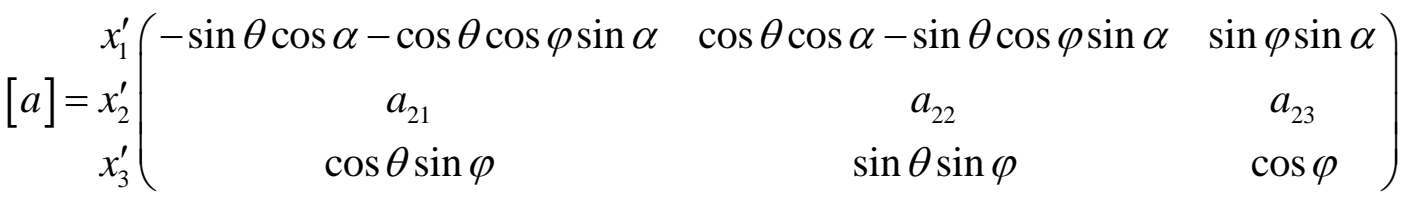

where, for the calculation under consideration, there is no need to determine analytical expressions for $a_{21}, a_{22}$ and $a_{23}$. The Poisson's ratio for a given loading direction as a function of $\alpha$ can now be obtained by using equations (2) and (3) in equation (1) as

$$
v_{m n}=v_{13}^{\prime}(\alpha)=-\frac{a_{1 p} a_{1 q} a_{3 r} a_{3 s} s_{p q r s}}{a_{3 p} a_{3 q} a_{3 r} a_{3 s} s_{p q r s}} .
$$

The $a_{i j}$ values are obtained using equation (7).

If Poisson's ratio is isotropic in the transverse plane when loaded along $\hat{\mathbf{n}}, v_{13}^{\prime}(\alpha)$ will be independent of $\alpha$. The denominator on the right-hand side in equation (8) is the reciprocal of the Young's modulus along $\widehat{\mathbf{n}}$ which is always greater than zero and is independent of $\alpha$. Therefore, $v_{13}^{\prime}$ is independent of $\alpha$ when $s_{13}^{\prime}$ is independent of $\alpha$. For the general (triclinic) case, the expression for $s_{13}^{\prime}$ can be simplified down to an equation of the form

$$
s_{13}^{\prime}=A\left(s_{i j}, \theta, \varphi\right) \sin \alpha \cos \alpha+B\left(s_{i j}, \theta, \varphi\right) \sin ^{2} \alpha+C\left(s_{i j}, \theta, \varphi\right) .
$$

Since $A$ and $B$ are functions of $s_{i j}$ and of the angular coordinates of the loading direction alone, $s_{13}^{\prime}$ will be independent of $\alpha$ when

$$
A=B=0
$$

The values of the compliance coefficients for all the materials used in this study are shown in Tables 1, 2 and 3. These values in Tables 1, 2 and 3 obtained from experimental measurements have been taken to be exact, so that the results of our calculations can be confirmed. It is to be noted that these experimental measurements were carried out at nominally identical thermodynamic conditions of room temperature and atmospheric pressure. 


\subsection{Representation of Loading Directions for which Poisson's Ratio is}

\section{Isotropic in the Transverse Plane}

A three-dimensional (3D) geometrical representation of the directional dependence of Poisson's ratio is not straightforward because it involves two directions - a loading direction and a transverse direction. An existing method to visualize the anisotropy of Poisson's ratio in 3D uses two surfaces (Marmier et al, 2010): one each through the maximum and minimum values of Poisson's ratio along each loading direction. The surface representing the minimum values is made opaque and is enveloped by the surface representing the maximum values, which is made translucent. Loading directions for which Poisson's ratio is isotropic in the transverse plane will be the directions along which the maximum and minimum surfaces coincide. This can be useful in representing the extreme Poisson's ratios for each loading direction, but it does not clearly highlight the loading directions for which Poisson's ratio is isotropic in the transverse plane.

In the current study, a new surface to visualise the anisotropy of Poisson's ratio in 3D has been developed. This surface will be referred to as an "anisotropy surface". For this surface, the radius vector lies along the loading direction $\hat{\mathbf{n}}$. Its magnitude is defined as

$$
r(\hat{\mathbf{n}})=K-\left[\max \left(v_{m n}\right)-\min \left(v_{m n}\right)\right]
$$

where $K$ is defined as the maximum possible difference in Poisson's ratio in the transverse plane, i.e., the $\max \left(v_{m n}\right)-\min \left(v_{m n}\right)$, for the loading direction in the material for which Poisson's ratio is most anisotropic in the transverse plane. The advantage of this surface is that it clearly depicts the extent of anisotropy along each loading direction. Loading directions for which Poisson's ratio is exactly, or nearly, isotropic in the transverse plane will appear as peaks of this surface. By contrast, the surface converges to the origin for those loading directions for which Poisson's ratio is most anisotropic in the transverse plane. 
The construction of this surface is explained using copper as an example. In copper, and indeed the overwhelming majority of cubic materials, the maximum difference in Poisson's ratio in the plane transverse to the loading direction occurs when the loading is along $\langle 110\rangle_{V}$. Exceptions such as the cubic materials $\mathrm{Al}-60 \mathrm{at} \% \mathrm{Ni}$ at $273 \mathrm{~K}, \mathrm{Cu}-14.5 \mathrm{wt} \% \mathrm{Al}-3.15 \mathrm{wt} \% \mathrm{Ni}$ at room temperature and $\mathrm{In}-27$ at $\% \mathrm{Tl}$ at $200 \mathrm{~K}$ where the maximum difference in Poisson's ratio in the plane transverse to the loading direction is not along $\langle 110\rangle_{V}$ are discussed by Norris (2006b). Using the compliance values for copper in Table 1, the variation of Poisson's ratio in the transverse plane for $[110]_{V}$ loading in copper is shown in Figure 3. The maximum Poisson's ratio is 0.820 along $[001]_{V}$ and the minimum is -0.133 along $[1 \overline{1} 0]_{V}$ so that $K=$ $0.820-(-0.133)=0.953$. For $[112]_{V}$ loading (Figure 3$)$, the maximum Poisson's ratio in the transverse plane is 0.502 along $[1 \overline{1} 0]_{V}$ and the minimum value is 0.184 along $[111]_{V}$. Therefore, $r\left([112]_{V}\right)=0.953-(0.502-0.184)=0.635$. Similarly, the length of the radius vector is calculated for each of the loading directions and the surface is plotted (Figure 4). A peak would represent a loading direction for which Poisson's ratio is isotropic in the transverse plane only if the radius along the corresponding loading direction is equal to $K$. Therefore this value has to be known when visualising the anisotropy surface. The $K$ value gives the upper limit of anisotropy along the transverse plane for a given material. All the anisotropy surfaces in this study were plotted using GNU Octave (Eaton, et al. 2016).

The loading directions for which Poisson's ratio is isotropic in the transverse plane can also be represented by plotting them on a stereogram centred on 001. The stereogram showing these loading directions marked by the letters A-I on the anisotropy surface in copper are shown in Figure 4. The stereogram has the advantage that it can depict all the loading directions for which Poisson's ratio is isotropic in the transverse plane in a way that is easily decipherable. However, it can only represent these loading directions and does not give any information about the degree of anisotropy along other directions. 


\section{Results and Discussion}

For the general case, i.e., for triclinic crystals,

$$
\begin{aligned}
A & =\frac{1}{16}\left\{\left[\left(11 s_{14}+s_{24}+4 s_{34}-5 s_{56}\right) \cos \theta+\left(s_{14}-s_{24}+s_{56}\right) \cos 3 \theta-\left(s_{15}+11 s_{25}+4 s_{35}-5 s_{46}\right) \sin \theta\right.\right. \\
& \left.+\left(-s_{15}+s_{25}+s_{46}\right) \sin 3 \theta\right] \sin \phi+4 \cos \phi\left[\operatorname { c o s } 2 \theta \left(-s_{16}-s_{26}-2 s_{36}+2 s_{45}\right.\right. \\
& \left.+\left[s_{16}+s_{26}-2\left(s_{36}+s_{45}\right)\right] \cos 2 \phi\right)+\left(s_{11}+2 s_{13}-s_{22}-2 s_{23}+s_{44}-s_{55}\right. \\
& \left.+\left(-s_{11}+2 s_{13}+s_{22}-2 s_{23}-s_{44}+s_{55}\right) \cos 2 \phi\right) \sin 2 \theta \\
& \left.+\left(2\left[-s_{16}+s_{26}\right] \cos 4 \theta+\left[s_{11}-2 s_{12}+s_{22}-s_{66}\right] \sin 4 \theta\right) \sin ^{2} \phi\right]-\left[\left(s_{14}+3 s_{24}-4 s_{34}+s_{56}\right) \cos \theta\right. \\
& \left.\left.+3\left(s_{14}-s_{24}+s_{56}\right) \cos 3 \theta-\left(3 s_{15}+s_{25}-4 s_{35}+s_{46}\right) \sin \theta+3\left(-s_{15}+s_{25}+s_{46}\right) \sin 3 \theta\right] \sin 3 \phi\right\}
\end{aligned}
$$

and

$$
\begin{aligned}
B & =\frac{1}{64}\left\{-\left[3 s_{11}+2 s_{12}-8 s_{13}+3 s_{22}-4\left(2 s_{23}-2 s_{33}+s_{44}+s_{55}\right)+s_{66}\right] \cos 4 \phi\right. \\
& +4\left[s_{16}+s_{26}+10 s_{36}-2 s_{45}-\left(s_{16}+s_{26}-2\left[s_{36}+s_{45}\right]\right) \cos 4 \phi\right] \sin 2 \theta \\
& +4 \cos 2 \theta\left[s_{11}+10 s_{13}-s_{22}-10 s_{23}+s_{44}-s_{55}+4\left(s_{13}-s_{23}\right) \cos 2 \phi\right. \\
& \left.+\cos 4 \phi\left(-s_{11}+2 s_{13}+s_{22}-2 s_{23}-s_{44}+s_{55}+\left[-s_{16}+s_{26}\right] \sin 2 \theta\right)\right] \\
& +10\left(s_{16}-s_{26}\right) \sin 4 \theta+32\left(s_{15}-s_{25}-s_{46}\right) \cos 3 \theta \cos ^{3} \phi \sin \phi \\
& +32\left(s_{14}-s_{24}+s_{56}\right) \cos ^{3} \phi \sin 3 \theta \sin \phi+12\left(s_{11}-2 s_{12}+s_{22}-s_{66}\right) \cos 4 \theta \sin ^{2} \phi \\
& +4 \cos 2 \phi\left[s_{11}+6 s_{12}-4 s_{13}+s_{22}-4 s_{23}-s_{66}+4 s_{36} \sin 2 \theta\right. \\
& \left.+2\left(-s_{16}+s_{26}\right) \sin 4 \theta+\left(s_{11}-2 s_{12}+s_{22}-s_{66}\right) \cos 4 \theta \sin { }^{2} \phi\right] \\
& +8\left[-3 s_{14}-s_{24}+s_{56}+\left(s_{14}+3 s_{24}-4 s_{34}+s_{56}\right) \cos 2 \phi\right] \sin \theta \sin 2 \phi \\
& +4 \cos \theta\left[-2\left(s_{15}+3 s_{25}-s_{46}\right) \sin 2 \phi+\left(3 s_{15}+s_{25}-4 s_{35}+s_{46}\right) \sin 4 \phi\right] \\
& \left.-\left[s_{11}+22 s_{12}-8 s_{13}+s_{22}-8 s_{23}-8 s_{33}+4\left(s_{44}+s_{55}\right)-5 s_{66}\right]\right\}
\end{aligned}
$$

in equation (10). Equations (12) and (13) will reduce to simpler forms for point groups with fewer independent compliances. These cases are discussed in the following subsections.

\subsection{The Cubic System}

Here, the Voigt compliance matrix $s_{i j}$ takes the form (Nye, 1957) given below: 


$$
s_{i j}=\left(\begin{array}{cccccc}
s_{11} & s_{12} & s_{12} & 0 & 0 & 0 \\
s_{12} & s_{11} & s_{12} & 0 & 0 & 0 \\
s_{12} & s_{12} & s_{11} & 0 & 0 & 0 \\
0 & 0 & 0 & s_{44} & 0 & 0 \\
0 & 0 & 0 & 0 & s_{44} & 0 \\
0 & 0 & 0 & 0 & 0 & s_{44}
\end{array}\right) .
$$

Using these compliances, equations (12) and (13) reduce to the expressions:

$$
A=\frac{1}{4}\left(2 s_{11}-2 s_{12}-s_{44}\right) \cos \phi \sin 4 \theta \sin ^{2} \phi
$$

and

$$
B=\frac{1}{16}\left(2 s_{11}-2 s_{12}-s_{44}\right)[5+7 \cos 2 \phi+\cos 4 \theta(3+\cos 2 \phi)] \sin ^{2} \phi
$$

One invariant solution independent of the compliance constants of equation (10) occurs when $\varphi=0^{\circ}$. This is the cube axis $[001]_{V}$. From the symmetry of cubic crystals, the other cube axes $[100]_{V}$ and $[010]_{V}$ will also be loading directions for which Poisson's ratio is isotropic in the transverse plane. Another solution is when $\theta=45^{\circ}$ and $\cos \varphi=1 / \sqrt{3}$. This defines one of the $<111>$ body diagonals of the cube. Therefore, by symmetry, all the body diagonals of the cube are loading directions for which Poisson's ratio is isotropic in the transverse plane.

A further possibility for isotropy of Poisson's ratio is when $2\left(s_{11}-s_{12}\right)=s_{44}$. In this case, the material will be completely isotropic. This is also the condition for complete isotropy of Young's modulus (Nye, 1957). An example of a cubic material whose compliance coefficients (Table 1) satisfy this condition is tungsten $(m \overline{3} m)$.

It is therefore seen that a cubic crystal, independent of its compliance values, has two types of loading directions for which Poisson's ratio is isotropic in the transverse plane: the cube axes, $\langle 100\rangle_{V}$, and the body diagonals, $\langle 111\rangle_{V}$. The body diagonals are three-fold axes for all the five cubic point groups. However, the cube axes can be either two-fold (point 
groups 23 and $m \overline{3})$ or four-fold $(m \overline{3} m, 432$ and $4 \overline{3} m)$. Cubic crystals also have two-fold axes along the face diagonals, $\langle 110\rangle_{V}$. These directions are not loading directions for which Poisson's ratio is isotropic in the transverse plane. It can therefore be concluded that the three-fold and four-fold axes in cubic crystals are loading directions for which Poisson's ratio is isotropic in the transverse plane. However, the two-fold axes along $\langle 100\rangle_{V}$ satisfy this condition but those along $\langle 110\rangle_{V}$ do not. The values of Poisson's ratio along each of the loading directions in copper and tungsten for which Poisson's ratio is isotropic in the transverse plane are shown in Table 4.

\section{EXAMPLE}

The anisotropy surface for copper $(m \overline{3} m)$ is shown in Figure 4 . The $K$ value is 0.95363 . The peaks along the cube axes are broader than those along the body diagonals showing that the departure from isotropy of Poisson's ratio within the transverse plane increases faster as the loading direction is moved away from a body diagonal than when it is moved away from a cube axis.

\subsection{The Tetragonal System}

Depending on the point group, there are two possible forms of the compliance matrix for tetragonal materials. Each of these cases is discussed separately.

\subsubsection{Point Groups $4, \overline{4}$ and $4 / m$}

The compliance tensors for materials with point group classes $4, \overline{4}$ and $4 / m$ have seven independent components. They are of the form (Nye, 1957):

$$
s_{i j}=\left(\begin{array}{cccccc}
s_{11} & s_{12} & s_{13} & 0 & 0 & s_{16} \\
s_{12} & s_{11} & s_{13} & 0 & 0 & -s_{16} \\
s_{13} & s_{13} & s_{33} & 0 & 0 & 0 \\
0 & 0 & 0 & s_{44} & 0 & 0 \\
0 & 0 & 0 & 0 & s_{44} & 0 \\
s_{16} & -s_{16} & 0 & 0 & 0 & s_{66}
\end{array}\right) .
$$


Therefore, for these materials, equations (12) and (13) become:

$$
A=\frac{1}{4} \sin ^{2} \phi \cos \phi\left[-4 s_{16} \cos 4 \theta+\left(2 s_{11}-2 s_{12}-s_{66}\right) \sin 4 \theta\right]
$$

and

$$
\begin{aligned}
B= & \frac{1}{16} \sin ^{2} \phi\left[2 s_{11}-10 s_{12}+8 s_{33}-8 s_{44}+3 s_{66}+\left(2 s_{11}-2 s_{12}-s_{66}\right) \cos 4 \theta(3+\cos 2 \phi)\right. \\
& \left.+12 s_{16} \sin 4 \theta+\cos 2 \phi\left(6 s_{11}+2 s_{12}-16 s_{13}+8 s_{33}-8 s_{44}+s_{66}+4 s_{16} \sin 4 \theta\right)\right] .
\end{aligned}
$$

It is immediately apparent from equations (16) and (17) that equation (10), the condition that $A=B=0$, has a solution when $\varphi=0^{\circ}$, i.e., when the loading direction is along the four-fold axis. The other solutions are dependent on the compliance coefficients of the material.

$A=0$ in equation (16) when $\varphi=90^{\circ}$. These are directions that lie in the $\left(x_{1}, x_{2}\right)$ basal plane of the crystal. These directions will repeat after a $90^{\circ}$ rotation about $[001]_{V}$. The solutions of $B=0$ in equation (17) are given by

$$
\theta=\frac{1}{2} \tan ^{-1}\left(\frac{-c \pm \sqrt{c^{2}+b^{2}-a^{2}}}{a-b}\right)
$$

where

$$
\begin{aligned}
& a=-2 s_{11}-6 s_{12}+8 s_{13}+s_{66} \\
& b=2 s_{11}-2 s_{12}-s_{66} \\
& c=4 s_{16} .
\end{aligned}
$$

Therefore, there can be a maximum of two distinct families of loading directions lying in the basal plane for materials with point groups $4, \overline{4}$ or $4 / m$ for which Poisson's ratio is isotropic in the transverse plane.

\section{EXAMPLE}

All possible families of loading directions in calcium molybdate $\left(\mathrm{CaMoO}_{4}\right)$, a mineral with scheelite structure $(4 / m)$, for which Poisson's ratio is isotropic in the transverse plane are 
shown in Table 5. The anisotropy surface for calcium molybdate (Figure 5) has ten peaks, corresponding to three distinct families of such loading directions. The two peaks along the four-fold axis are much broader than the eight other peaks. Therefore, the departure from isotropy of Poisson's ratio within the transverse plane is lower as the loading axis moves away from the four-fold axis, than it is for those around the compliance-dependent loading directions. Since $K$ for calcium molybdate is 0.32617 , Poisson's ratio in calcium molybdate is anisotropic, but less so than copper.

\subsubsection{Point Groups 422, $4 \mathrm{~mm}, \overline{4} 2 \mathrm{~m}$ and $4 / \mathrm{mmm}$}

The compliance matrix for these tetragonal point groups has six independent constants. It is of the form (Nye, 1957):

$$
s_{i j}=\left(\begin{array}{cccccc}
s_{11} & s_{12} & s_{13} & 0 & 0 & 0 \\
s_{12} & s_{11} & s_{13} & 0 & 0 & 0 \\
s_{13} & s_{13} & s_{33} & 0 & 0 & 0 \\
0 & 0 & 0 & s_{44} & 0 & 0 \\
0 & 0 & 0 & 0 & s_{44} & 0 \\
0 & 0 & 0 & 0 & 0 & s_{66}
\end{array}\right) .
$$

Therefore, for materials in these crystal classes, equations (12) and (13) become:

$$
A=\frac{1}{4} \cos \phi \sin 4 \theta \sin ^{2} \phi\left(2 s_{11}-2 s_{12}-s_{66}\right)
$$

and

$$
\begin{aligned}
B= & \frac{1}{16} \sin ^{2} \phi\left[2 s_{11}-10 s_{12}+8 s_{33}-8 s_{44}+3 s_{66}+\left(2 s_{11}-2 s_{12}-s_{66}\right) \cos 4 \theta(3+\cos 2 \phi)\right. \\
& \left.+\cos 2 \phi\left(6 s_{11}+2 s_{12}-16 s_{13}+8 s_{33}-8 s_{44}+s_{66}\right)\right]
\end{aligned}
$$

Here too, the four-fold axis (when $\varphi=0^{\circ}$ ) is a loading direction for which Poisson's ratio is isotropic in the transverse plane. Therefore, this is a general result for all tetragonal materials. 
A solution to $A=0$ in equation (18) occurs when $\varphi=90^{\circ}$. Under these circumstances $\theta=\frac{1}{4} \cos ^{-1}\left(-\frac{-2 s_{11}-6 s_{12}+8 s_{13}+s_{66}}{2 s_{11}-2 s_{12}-s_{66}}\right)$ will satisfy $B=0$ in equation (19). There can be a maximum of one such family of loading directions. Another solution to $A=0$ in equation (18) is when $\theta=0^{\circ}$ and $\theta=45^{\circ}$ for $0^{\circ} \leq \theta<90^{\circ}$. When $\theta=0^{\circ}$, the solution to $B=0$ in equation (19) is

$$
\varphi=\frac{1}{2} \cos ^{-1}\left(-\frac{s_{11}-2 s_{12}+s_{33}-s_{44}}{s_{11}-2 s_{13}+s_{33}-s_{44}}\right) .
$$

When $\theta=45^{\circ}, B=0$ in equation (19) is satisfied by

$$
\varphi=\frac{1}{2} \cos ^{-1}\left(\frac{2 s_{11}+2 s_{12}-4 s_{33}+4 s_{44}-3 s_{66}}{2 s_{11}+2 s_{12}-8 s_{13}+4 s_{33}-4 s_{44}+s_{66}}\right) .
$$

\section{EXAMPLE}

The anisotropy surface for indium $(4 / \mathrm{mmm})$ is shown in Figure 6 . The $K$ value is 1.7315 , suggesting that the Poisson's ratio in indium can be highly anisotropic. Such high values of $K$ can be expected for auxetic materials. In spite of such a significant anisotropy, the anisotropy surface for indium has eighteen peaks with $r=K$ corresponding to three distinct families of loading directions for which Poisson's ratio is isotropic in the transverse plane (Table 6). This surface has eight peaks each corresponding to $\theta=0^{\circ}$ and $\theta=45^{\circ}$ and two peaks for $\varphi=0^{\circ}$. However, there are no solutions for $\varphi=90^{\circ}$ (i.e., for loading directions lying in the basal plane) because the argument of $\cos ^{-1}$ in the expression for $\theta$ discussed above is less than -1 . It is evident that the peaks along the four-fold axis are broader than the other peaks.

\subsection{The Hexagonal System}

The elastic properties of materials with hexagonal symmetry are transversely isotropic. The compliance matrix is of the form (Nye, 1957): 


$$
s_{i j}=\left(\begin{array}{cccccc}
s_{11} & s_{12} & s_{13} & 0 & 0 & 0 \\
s_{12} & s_{11} & s_{13} & 0 & 0 & 0 \\
s_{13} & s_{13} & s_{33} & 0 & 0 & 0 \\
0 & 0 & 0 & s_{44} & 0 & 0 \\
0 & 0 & 0 & 0 & s_{44} & 0 \\
0 & 0 & 0 & 0 & 0 & 2\left(s_{11}-s_{12}\right)
\end{array}\right) .
$$

With these compliances in equation (12), $A$ is equal to zero. $B$ in equation (13) takes the form

$$
B=\frac{1}{2}\left[s_{11}-2 s_{12}+s_{33}-s_{44}+\left(s_{11}-2 s_{13}+s_{33}-s_{44}\right) \cos 2 \phi\right] \sin ^{2} \phi
$$

It is evident that $\varphi=0^{\circ}$ is a solution to $B=0$. This shows that the six-fold axis is a loading direction in all hexagonal materials for which Poisson's ratio is isotropic in the transverse plane. Furthermore, since $B$ is dependent on $\varphi$ alone, the compliance-dependent loading directions of such kind, if present, will be infinitely many directions at a fixed inclination given by $\varphi=\frac{1}{2} \cos ^{-1}\left(-\frac{s_{11}+s_{33}-s_{44}-2 s_{12}}{s_{11}+s_{33}-s_{44}-2 s_{13}}\right)$.

\section{EXAMPLE}

The anisotropy surface for zinc $(6 / \mathrm{mmm})$ is shown in Figure 7 . There are peaks along the sixfold axis. The compliance-dependent directions for which Poisson's ratio is isotropic in the transverse plane lie along the surface of a right circular cone with its apex at the origin, its axis along the six-fold axis and a semi-apex angle as given above. The peaks along the sixfold axis are broader than the compliance-dependent peaks. It can also be observed that the directions in the basal plane of zinc are the loading directions for which Poisson's ratio is most anisotropic in the transverse plane. The anisotropy of Poisson's ratio in zinc is significant because the $K$ value is 0.92373 .

\subsection{The Orthorhombic System}

The compliance matrix for orthorhombic materials is of the form (Nye, 1957): 


$$
s_{i j}^{\prime}=\left(\begin{array}{cccccc}
s_{11} & s_{12} & s_{13} & 0 & 0 & 0 \\
s_{12} & s_{22} & s_{23} & 0 & 0 & 0 \\
s_{13} & s_{23} & s_{33} & 0 & 0 & 0 \\
0 & 0 & 0 & s_{44} & 0 & 0 \\
0 & 0 & 0 & 0 & s_{55} & 0 \\
0 & 0 & 0 & 0 & 0 & s_{66}
\end{array}\right) .
$$

Hence, in equations (12) and (13),

$$
\begin{aligned}
A= & \frac{1}{4} \cos \varphi\left\{\left[s_{11}+2 s_{13}-s_{22}-2 s_{23}+s_{44}-s_{55}+\left(-s_{11}+2 s_{13}+s_{22}-2 s_{23}\right.\right.\right. \\
& \left.\left.\left.-s_{44}+s_{55}\right) \cos 2 \varphi\right] \sin 2 \theta+\left(s_{11}-2 s_{12}+s_{22}-s_{66}\right) \sin 4 \theta \sin ^{2} \varphi\right\}
\end{aligned}
$$

and

$$
\begin{aligned}
B & =\frac{1}{16}\left\{\operatorname { c o s } 2 \theta \left[s_{11}+10 s_{13}-s_{22}-10 s_{23}+s_{44}-s_{55}+4\left(s_{13}-s_{23}\right) \cos 2 \varphi\right.\right. \\
& \left.+\left(-s_{11}+2 s_{13}+s_{22}-2 s_{23}-s_{44}+s_{55}\right) \cos 4 \varphi\right]+\sin ^{2} \varphi\left[s_{11}-10 s_{12}+s_{22}+8 s_{33}\right. \\
& -4\left(s_{44}+s_{55}\right)+3 s_{66}+\left(3 s_{11}+2 s_{12}-8 s_{13}+3 s_{22}-4\left(2 s_{23}-2 s_{33}+s_{44}+s_{55}\right)+s_{66}\right) \cos 2 \varphi \\
& \left.\left.+\left(s_{11}-2 s_{12}+s_{22}-s_{66}\right) \cos 4 \theta(3+\cos 2 \varphi)\right]\right\}
\end{aligned}
$$

One solution for $A=0$ in equation (21) occurs when $\varphi=90^{\circ}$ for $0^{\circ} \leq \varphi \leq 90^{\circ}$. For this solution, $B=0$ in equation (22) when $\theta=\frac{1}{2} \cos ^{-1}\left(\frac{-b \pm \sqrt{b^{2}-4 a c}}{2 a}\right)$, where $a, b$ and $c$ are defined in Row 1 of Table 7. This gives a maximum of two families of loading directions for which Poisson's ratio is isotropic in the transverse plane.

Another solution for $A=0$ in equation (21) occurs when $\theta=0^{\circ}$ or $\theta=90^{\circ}$. For the case where $\theta=0^{\circ}, B=0$ in equation (22) when $\varphi=\frac{1}{2} \cos ^{-1}\left(\frac{-b \pm \sqrt{b^{2}-4 a c}}{2 a}\right)$ where $a, b$ and $c$ are defined in Row 2 of Table 7. When $\theta=90^{\circ}, \varphi=\frac{1}{2} \cos ^{-1}\left(\frac{-b \pm \sqrt{b^{2}-4 a c}}{2 a}\right)$ where $a, b$ and $c$ are defined in Row 3 of Table 7. 
A third solution when $A=0$ in equation (21) arises when

$\varphi=\sin ^{-1}\left( \pm \sqrt{\frac{2\left(s_{23}-s_{13}\right)}{s_{11}-2 s_{13}-s_{22}+2 s_{23}+s_{44}-s_{55}+\left(s_{11}-2 s_{12}+s_{22}-s_{66}\right) \cos 2 \theta}}\right)$

and

$\theta=\frac{1}{2} \cos ^{-1}(y)$, where $y=-\frac{q_{1}}{q_{2}}$, and where $q_{1}$ and $q_{2}$ are defined below based on the constants $p_{i}$ ( $i$ varies from 1 to 9 ) which are defined subsequently:

$$
\begin{gathered}
q_{1}=p_{7}\left[2\left(p_{6}-p_{5}\right) p_{7}+\left(p_{4}+p_{5}-4 p_{6}\right) p_{8}\right] \\
q_{2}=p_{7}\left[p_{9}\left(p_{5}+p_{4}-4 p_{6}\right)+8 p_{3} p_{7}-2\left(p_{2}+4 p_{3}\right) p_{8}\right]+\left(p_{1}+p_{2}+p_{3}\right) p_{8}^{2} \\
p_{1}=s_{11}+10 s_{13}-s_{22}-10 s_{23}+s_{44}-s_{55} \\
p_{2}=4\left(s_{13}-s_{23}\right) \\
p_{3}=-s_{11}+2 s_{13}+s_{22}-2 s_{23}-s_{44}+s_{55} \\
p_{4}=s_{11}-10 s_{12}+s_{22}+8 s_{33}-4\left(s_{44}+s_{55}\right)+3 s_{66} \\
p_{5}=3 s_{11}+2 s_{12}-8 s_{13}+3 s_{22}-4\left(2 s_{23}-2 s_{33}+s_{44}+s_{55}\right)+s_{66} \\
p_{6}=s_{11}-2 s_{12}+s_{22}-s_{66} \\
p_{8}=-\left(-s_{11}+2 s_{13}+s_{22}-2 s_{23}-s_{44}+s_{55}\right) \\
p_{7}=2\left(s_{23}-s_{13}\right) \\
s_{11}-2 s_{12}+s_{22}-s_{66} .
\end{gathered}
$$

\section{EXAMPLE}

All the possible loading directions for which Poisson's ratio is isotropic in the transverse plane in forsterite $(\mathrm{mmm})$ are shown in Table 8 . The anisotropy surface for forsterite (Figure 8) has twenty peaks, corresponding to four distinct families of loading directions for 
which Poisson's ratio is isotropic in the transverse plane. The value of $K$ is 0.18482 which suggests that the Poisson's ratio can vary less in the transverse plane than it does in copper, zinc and calcium molybdate.

\subsection{The Trigonal System}

Depending on the point group, there are two possible forms of the compliance matrix for trigonal materials. Each of these cases is discussed separately.

\subsubsection{Point Groups 3 and $\overline{3}$}

The compliance matrix for materials with point groups 3 and $\overline{3}$ is of the form (Nye, 1957):

$$
s_{i j}=\left(\begin{array}{cccccc}
s_{11} & s_{12} & s_{13} & s_{14} & s_{15} & 0 \\
s_{12} & s_{11} & s_{13} & -s_{14} & -s_{15} & 0 \\
s_{13} & s_{13} & s_{33} & 0 & 0 & 0 \\
s_{14} & -S_{14} & 0 & s_{44} & 0 & -2 s_{15} \\
s_{15} & -s_{15} & 0 & 0 & s_{44} & 2 s_{14} \\
0 & 0 & 0 & -2 s_{15} & 2 s_{14} & 2\left(s_{11}-s_{12}\right)
\end{array}\right) \text {. }
$$

with $[100]_{V}$ parallel to the crystal $x$-axis. Here, equations (12) and (13) simplify to:

$$
A=-\frac{1}{2} \sin \varphi(1+3 \cos 2 \varphi)\left(s_{14} \cos 3 \theta-s_{15} \sin 3 \theta\right)
$$

and

$$
\begin{aligned}
B= & \frac{1}{2} \sin \varphi\left[4 \cos ^{3} \varphi\left(s_{15} \cos 3 \theta+s_{14} \sin 3 \theta\right)\right. \\
& \left.+\left(s_{11}-2 s_{12}+s_{33}-s_{44}+\left(s_{11}-2 s_{13}+s_{33}-s_{44}\right) \cos 2 \varphi\right) \sin \varphi\right]
\end{aligned}
$$

$\varphi=0^{\circ}$ is a solution which gives $A=0$ and $B=0$ in equations (23) and (24) respectively. This proves that $[001]_{V}$, the three-fold axis, is always a loading direction for which Poisson's ratio is isotropic in the transverse plane in materials with point groups 3 and $\overline{3}$. 
Other solutions to $A=0$ are given by $\theta_{j}=\frac{1}{3} \tan ^{-1}\left(\frac{s_{14}}{s_{15}}\right)+j \pi$ where $j=0$ and 1 . For these solutions, $B=0$ for $\varphi_{k}=\tan ^{-1}\left\{2 \sqrt{\frac{-P}{3}} \cos \left[\frac{1}{3} \cos ^{-1}\left(\frac{3 Q}{2 P} \sqrt{\frac{-3}{P}}\right)-\frac{2 \pi k}{3}\right]\right\}$ where $k=0,1$ and 2, $P=\frac{p+q}{p-q}$ and $Q=\frac{r}{p-q}$, and where $p, q$ and $\mathrm{r}$ are given by the expressions:

$$
\begin{aligned}
& p=s_{11}-2 s_{12}+s_{33}-s_{44} \\
& q=s_{11}-2 s_{13}+s_{33}-s_{44} \\
& r=4\left(s_{15} \cos 3 \theta_{j}+s_{14} \sin 3 \theta_{j}\right) .
\end{aligned}
$$

\section{EXAMPLE}

The four families of loading directions in the ilmenite phase of magnesium silicate $\left(\mathrm{MgSiO}_{3}\right.$, point group $\overline{3}$ ) for which Poisson's ratio is isotropic in the transverse plane are shown in Table 9. The anisotropy surface of ilmenite (Figure 9) has twenty peaks. In contrast to copper, calcium molybdate, indium and zinc, the peaks along the compliance-dependent loading directions in $\mathrm{MgSiO}_{3}$ for which Poisson's ratio is isotropic in the transverse plane are broader than those along the three-fold axis. Therefore, it is not the case in general that loading directions lying close to the symmetry axes are more isotropic in their Poisson's ratio behaviour than the directions lying close to the compliance-dependent loading directions with exact isotropic Poisson's ratio behaviour. The $K$ value of $\mathrm{MgSiO}_{3}$ is 0.66229 and so the degree of anisotropy in Poisson's ratio can be significant.

\subsubsection{Point Groups 32, $\overline{3} m$ and $3 m$}

The compliance matrix for materials with these point groups is of the form (Nye, 1957): 


$$
s_{i j}=\left(\begin{array}{cccccc}
s_{11} & s_{12} & s_{13} & s_{14} & 0 & 0 \\
s_{12} & s_{11} & s_{13} & -s_{14} & 0 & 0 \\
s_{13} & s_{13} & s_{33} & 0 & 0 & 0 \\
s_{14} & -s_{14} & 0 & s_{44} & 0 & 0 \\
0 & 0 & 0 & 0 & s_{44} & 2 s_{14} \\
0 & 0 & 0 & 0 & 2 s_{14} & 2\left(s_{11}-s_{12}\right)
\end{array}\right) .
$$

with $[100]_{V}$ parallel to the crystal $x$-axis. This can be obtained by setting $s_{15}=0$ in the compliance matrix for the point groups 3 and $\overline{3}$ discussed in Section 3.5.1. Substituting $s_{15}=$ 0 in equations (23) and (24),

$$
A=-\frac{s_{14}}{2}(1+3 \cos 2 \varphi) \cos 3 \theta \sin \varphi
$$

and

$$
\begin{aligned}
B= & \frac{1}{2} \sin \varphi\left[4 s_{14} \cos ^{3} \varphi \sin 3 \theta+\left(s_{11}-2 s_{12}+s_{33}-s_{44}\right.\right. \\
& \left.\left.+\left(s_{11}-2 s_{13}+s_{33}-s_{44}\right) \cos 2 \varphi\right) \sin \varphi\right] .
\end{aligned}
$$

One solution for $A=B=0$ in equations (25) and (26) is $\varphi=0$. This shows that the three-fold axis is always a loading direction for which Poisson's ratio is isotropic in the transverse plane. Other solutions for $A=0$ occur when $\theta=30^{\circ}, 90^{\circ}, 150^{\circ}, 210^{\circ}, 270^{\circ}$ or $330^{\circ}$. For these solutions, $B=0$ when $\varphi_{k}=\tan ^{-1}\left\{2 \sqrt{\frac{-P}{3}} \cos \left[\frac{1}{3} \cos ^{-1}\left(\frac{3 Q}{2 P} \sqrt{\frac{-3}{P}}\right)-\frac{2 \pi k}{3}\right]\right\}$, where $k=0$, 1 and $2, P=\frac{p+q}{p-q}$ and $Q=\frac{r}{p-q}$, where

$$
\begin{aligned}
& p=s_{11}-2 s_{12}+s_{33}-s_{44} \\
& q=s_{11}-2 s_{13}+s_{33}-s_{44} \\
& r=4 s_{14} \sin 3 \theta .
\end{aligned}
$$

\section{EXAMPLE}


$\alpha$-Quartz $\left(\mathrm{SiO}_{2}\right)$ is a mineral with the point group 32. The loading directions for which Poisson's ratio is isotropic in the transverse plane and the corresponding Poisson's ratios are shown in Table 10. There are two families of such loading directions, including the three-fold axis. Interestingly, the family of six loading directions inclined to the three-fold axis have Poisson's ratios which are negative. The anisotropy surface for $\alpha$-quartz (Figure 10) has eight peaks corresponding to these two families of loading directions. The $K$ value is 0.37937 , which means that Poisson's ratio can be significantly anisotropic within a plane transverse to a general loading direction in $\alpha$-quartz.

\subsection{The Monoclinic System}

The compliance matrix for monoclinic materials with the two-fold axis parallel to $x_{2}$ is of the form (Nye, 1957):

$$
s_{i j}=\left(\begin{array}{cccccc}
s_{11} & s_{12} & s_{13} & 0 & s_{15} & 0 \\
s_{12} & s_{22} & s_{23} & 0 & s_{25} & 0 \\
s_{13} & s_{23} & s_{33} & 0 & s_{35} & 0 \\
0 & 0 & 0 & s_{44} & 0 & s_{46} \\
s_{15} & s_{25} & s_{35} & 0 & s_{55} & 0 \\
0 & 0 & 0 & s_{46} & 0 & s_{66}
\end{array}\right) .
$$

Here, equations (12) and (13) simplify to:

$$
\begin{aligned}
A= & \frac{1}{16}\left\{\left[-\left(s_{15}+11 s_{25}+4 s_{35}-5 s_{46}\right) \sin \theta+\left(-s_{15}+s_{25}+s_{46}\right) \sin 3 \theta\right] \sin \phi\right. \\
& +4 \cos \phi\left[\left(s_{11}-2 s_{12}+s_{22}-s_{66}\right) \sin 4 \theta \sin ^{2} \phi\right. \\
& \left.+\left(s_{11}+2 s_{13}-s_{22}-2 s_{23}+s_{44}-s_{55}+\left(-s_{11}+2 s_{13}+s_{22}-2 s_{23}-s_{44}+s_{55}\right) \cos 2 \phi\right) \sin 2 \theta\right] \\
& \left.-\left[-\left(3 s_{15}+s_{25}-4 s_{35}+s_{46}\right) \sin \theta+3\left(-s_{15}+s_{25}+s_{46}\right) \sin 3 \theta\right] \sin 3 \phi\right\}
\end{aligned}
$$

and 


$$
\begin{aligned}
B & =\frac{1}{16}\left\{\operatorname { c o s } 2 \theta \left[s_{11}+10 s_{13}-s_{22}-10 s_{23}+s_{44}-s_{55}+4\left(s_{13}-s_{23}\right) \cos 2 \phi\right.\right. \\
& \left.+\left(-s_{11}+2 s_{13}+s_{22}-2 s_{23}-s_{44}+s_{55}\right) \cos 4 \phi\right]+8\left(s_{15}-s_{25}-s_{46}\right) \cos 3 \theta \cos ^{3} \phi \sin \phi \\
& +\left(s_{11}-2 s_{12}+s_{22}-s_{66}\right) \cos 4 \theta(3+\cos 2 \phi) \sin ^{2} \phi \\
& +\left[s_{11}-10 s_{12}+s_{22}+8 s_{33}-4\left(s_{44}+s_{55}\right)+3 s_{66}\right. \\
& \left.+\left(3 s_{11}+2 s_{12}-8 s_{13}+3 s_{22}-4\left(2 s_{23}-2 s_{33}+s_{44}+s_{55}\right)+s_{66}\right) \cos 2 \phi\right] \sin ^{2} \phi \\
& \left.+\cos \theta\left[-2\left(s_{15}+3 s_{25}-s_{46}\right) \sin 2 \phi+\left(3 s_{15}+s_{25}-4 s_{35}+s_{46}\right) \sin 4 \phi\right]\right\}
\end{aligned}
$$

Solutions of equations (27) and (28) arise when $\theta=0^{\circ}$. The condition that $B=0$ results in a quartic equation in $\tan \varphi$ which can be solved by the method described in Wolfram Mathworld (Weisstein, 2018). This requires defining the following terms:

$$
\begin{aligned}
& p=s_{11}-4 s_{12}+6 s_{13}-4 s_{23}+s_{33}-s_{55} \\
& q=4\left(s_{12}-s_{23}\right) \\
& r=-s_{11}+2 s_{13}-s_{33}+s_{55} \\
& t=-4 s_{25} \\
& u=2\left(s_{15}-s_{35}\right) \\
& a_{3}=\frac{2(t-2 u)}{p-q+r} \\
& a_{2}=\frac{2(p-3 r)}{p-q+r} \\
& a_{1}=\frac{2(t+2 u)}{p-q+r} \\
& a_{0}=\frac{p+q+r}{p-q+r} \\
& A_{1}=-a_{2} \\
& A_{2}=a_{1} a_{3}-4 a_{0} \\
& A_{3}=4 a_{2} a_{0}-a_{1}^{2}-a_{3}^{2} a_{0}
\end{aligned}
$$




$$
\begin{aligned}
& Q=\frac{A_{1}^{2}-3 A_{2}}{9} \\
& R_{1}=\frac{2 A_{1}^{3}-9 A_{1} A_{2}+27 A_{3}}{54}
\end{aligned}
$$

If $Q^{3}-R_{1}^{2}>0$, we define

$$
\begin{aligned}
& T=\cos ^{-1}\left(\frac{R_{1}}{\sqrt{Q^{3}}}\right) \\
& y_{j}=-2 \sqrt{Q} \cos \left(\frac{T+2(j-1) \pi}{3}\right)-\frac{A_{1}}{3}, \text { where } j=1,2,3 .
\end{aligned}
$$

If $Q^{3}-R_{1}^{2}<0$, we define

$$
y_{1}=-\operatorname{sgn}\left(R_{1}\right)\left(\left(\sqrt{R_{1}^{2}-Q^{3}}+\left|R_{1}\right|\right)^{1 / 3}+\frac{Q}{\left(\sqrt{R_{1}^{2}-Q^{3}}+\left|R_{1}\right|\right)^{1 / 3}}\right)-\frac{A_{1}}{3}
$$

$R$ is now defined based on the real values of $y_{j}$ obtained above.

$$
R=\sqrt{\frac{a_{3}^{2}}{4}-a_{2}+y_{j}}
$$

If $R \neq 0$,

$$
\begin{aligned}
& D=\sqrt{\frac{3 a_{3}^{2}}{4}-R^{2}-2 a_{2}+\frac{4 a_{3} a_{2}-8 a_{1}-a_{3}^{3}}{4 R}} \\
& E=\sqrt{\frac{3 a_{3}^{2}}{4}-R^{2}-2 a_{2}-\frac{4 a_{3} a_{2}-8 a_{1}-a_{3}^{3}}{4 R}}
\end{aligned}
$$

If $R=0$,

$$
\begin{aligned}
& D=\sqrt{\frac{3 a_{3}^{2}}{4}-2 a_{2}+2 \sqrt{y_{j}^{2}-4 a_{0}}} \\
& E=\sqrt{\frac{3 a_{3}^{2}}{4}-2 a_{2}-2 \sqrt{y_{j}^{2}-4 a_{0}}}
\end{aligned}
$$

The solutions are: 


$$
\begin{aligned}
& \varphi_{1}=\tan ^{-1}\left(-\frac{a_{3}}{4}+\frac{R}{2}+\frac{D}{2}\right) \\
& \varphi_{2}=\tan ^{-1}\left(-\frac{a_{3}}{4}+\frac{R}{2}-\frac{D}{2}\right) \\
& \varphi_{3}=\tan ^{-1}\left(-\frac{a_{3}}{4}-\frac{R}{2}+\frac{E}{2}\right) \\
& \varphi_{4}=\tan ^{-1}\left(-\frac{a_{3}}{4}-\frac{R}{2}-\frac{E}{2}\right)
\end{aligned}
$$

For a general $\theta$ and $\varphi$, the solutions can be obtained for $A=B=0$ for monoclinic materials by a trial-and-error numerical solution - there would appear to be no analytical solutions. For this trial-and-error approach, $A$ and $B$ were computed as a function of $\theta$ and $\varphi$ on a grid where $\theta$ and $\varphi$ were systematically altered by suitable angular intervals, typically $1-5^{\circ}$. This enabled regions of $\theta-\varphi$ space to be interrogated more closely to find solutions to the desired accuracy of $\theta$ and $\varphi$ for which $A=B=0$. A second method used which confirmed these results was to determine in an Excel spreadsheet the maximum and minimum values of Poisson's ratio as a function of orientation within the transverse plane when sampled at $1^{\circ}$ intervals for a particular loading direction defined by its $\theta$ and $\varphi$, and then to find orientations for which the difference between these maximum and minimum values was zero.

\section{EXAMPLE}

The solutions obtained for bismuth vanadate (point group $2 / m$ ) are shown in Table 11 . There are five distinct families of loading directions for which Poisson's ratio is isotropic in the transverse plane, four of which have $\theta=0^{\circ}$. As a consequence, the anisotropy surface (Figure 11) for bismuth vanadate has twelve peaks. The $K$ value is 0.49019 . Therefore, for a suitable choice of loading direction, bismuth vanadate can have a significantly anisotropic Poisson's ratio in the transverse plane. 


\subsection{The Triclinic System}

The compliance matrices for triclinic materials generally have all 21 constants (Nye, 1957). $A$ and $B$ are given by equations (12) and (13). Like the general $\theta$ and $\varphi$ case for monoclinic crystals, there would appear to be no analytical solutions of the equations $A=B=0$ which therefore have to be solved by one or both of the numerical methods described in sub-section 3.6.

\section{EXAMPLE}

The solutions obtained for copper sulphate pentahydrate (point group $\overline{1}$ ) by numerical methods are shown in Table 12. The anisotropy surface of copper sulphate pentahydrate is shown in Figure 12. There are six families of loading directions for which Poisson's ratio is isotropic in the transverse plane, resulting in twelve peaks for the anisotropy surface. The value of 0.71444 for $K$ means that the Poisson's ratio can be significantly anisotropic.

\section{Conclusions}

Formulae for predicting the loading directions for which Poisson's ratio is isotropic in the transverse plane in single crystalline materials for all seven crystal systems have been derived. Explicit analytical expressions for the indices of the loading directions with respect to a suitable orthonormal axis system fixed with respect to the crystallographic axes have been obtained for cubic, tetragonal, hexagonal, orthorhombic and trigonal materials, and for one possible set of solutions for monoclinic materials. Numerical methods have been used to obtain other solutions in monoclinic and triclinic materials. Of particular note in the results shown in Section 3 is the existence of orientations in $\alpha$-quartz where the Poisson's ratio is not only isotropic in the transverse plane, but auxetic.

Loading along a three-fold, four-fold or six-fold axis of a single crystal will always result in a Poisson's ratio which is isotropic in the transverse plane. The only cases of two-fold axes 
being loading directions for which Poisson's ratio is isotropic in the transverse plane occur for materials with the cubic point groups 23 and $m \overline{3}$ when loaded along the cube axes.

Loading directions for which Poisson's ratio is isotropic in the plane transverse have been identified in materials belonging to each of the seven crystal systems irrespective of the non-zero compliance values. Orthorhombic, monoclinic and triclinic materials can only possess compliance-dependent loading directions for which Poisson's ratio is isotropic in the transverse plane, i.e., such loading directions cannot be along rotational axes of symmetry.

The anisotropy surface highlights the loading directions for a given material for which Poisson's ratio which is isotropic in the transverse plane. In addition it also shows graphically the anisotropy of Poisson's ratio in the transverse plane for other loading directions. 


\section{List of Figures}

Figure 1 Schematics of elastic deformation of the cross-sections of two cylindrical specimens when loaded along their axes. (a) a circular cross-section remains circular only if the Poisson's ratio is isotropic in the transverse plane for the given loading direction. (b) a circular cross-section will not remain circular if the Poisson's ratio is not isotropic in the transverse plan. For clarity, the magnitudes of the changes for a real single crystal have been heavily exaggerated in these two schematics.

Figure 2 (a) The Voigt $\left(x_{1}, x_{2}, x_{3}\right)$ and Poisson's $\left(x_{1}^{\prime}, x_{2}^{\prime}, x_{3}^{\prime}\right)$ coordinate systems for a given loading direction ( $(\hat{\mathbf{n}})$ for a rod under a uniaxial stress. The Poisson's coordinate system can be defined such that the $x_{1}^{\prime}$ axis lies along each the of the directions in the plane normal to the given loading direction by rotating the intermediate coordinate system $x_{1}^{\beta}, x_{2}^{\beta}, x_{3}^{\beta}$ by an angle $\alpha$ about the $x_{3}^{\beta}$ axis. Hence, for a given loading direction, the Poisson's ratio along each of the transverse directions can be calculated using equation (8). (b) Directions in the $x_{1}, x_{2}$ plane. OP is the projection of the loading direction $\hat{\mathrm{n}}$ on the $x_{1}, x_{2}$ plane. (c) Directions in the plane perpendicular to the loading direction $\hat{n}$ (the transverse plane).

Figure 3 Variation of the Poisson's ratio of copper in the transverse plane when loaded along $\langle 110\rangle_{V},\langle 111\rangle_{V},\langle 112\rangle_{V}$ and $\langle 100\rangle_{V}$. The straight line showing the values of Poisson's ratio is along the meridional tangent of the loading direction.

Figure 4 Representation of: (a) the anisotropy surface for copper (point group $m \overline{3} m$ ); (b) the surface viewed along the $-x_{2}$ direction; (c) the surface viewed along the $-x_{3}$ direction; (d) the loading directions in copper for which Poisson's ratio is isotropic in the transverse plane, represented on a stereogram centred at $[001]_{V}$. The directions have been labelled as follows: $\mathrm{A}$ is $[100]_{V}, \mathrm{~B}$ is $[010]_{V}, \mathrm{C}$ is $[\overline{1} 00]_{V}, \mathrm{D}$ is $[0 \overline{1} 0]_{V}, \mathrm{E}$ is $[111]_{V}, \mathrm{~F}$ is $[\overline{1} 11]_{V}, \mathrm{G}$ is $[\overline{1} \overline{1} 1]_{V}, \mathrm{H}$ is $[1 \overline{1} 1]_{V}$ and $\mathrm{I}$ is $[001]_{V}$. For clarity, only loading directions in the northern hemisphere and on the equatorial plane have been specified in the stereogram in (d) and other stereograms in this paper.

Figure 5 Representation of: (a) the anisotropy surface of calcium molybdate (point group $4 / m$ ); (b) the view along the $-x_{1}$ direction; (c) the view along the $-x_{3}$ direction; (d) the loading directions in calcium molybdate for which Poisson's ratio is isotropic in the transverse plane, represented on a stereogram centred on $[001]_{V}$ showing all the directions marked from $\mathrm{A}-\mathrm{I}$ in (c). The directions have been labelled as follows: $\mathrm{A}$ is $[0.962155,-0.272503,0]_{V}, \mathrm{~B}$ is $[-0.272503$, $-0.962155,0]_{V}, \mathrm{C}$ is $[-0.962155,0.272503,0]_{V}, \mathrm{D}$ is $[0.272503,0.962155,0]_{V}$, $\mathrm{E}$ is $[0.496618,0.867969,0]_{V}, \quad \mathrm{~F}$ is $[0.867969,-0.496618,0]_{V}, \quad \mathrm{G}$ is $[-0.496618,-0.867969,0]_{V}, \mathrm{H}$ is $[-0.867969,0.496618,0]_{V}$ and $\mathrm{I}$ is $[001]_{V}$.

Figure 6 Representation of: (a) the anisotropy surface of indium (point group 4/ $\mathrm{mmm}$ ); (b) the view along the $-x_{1}$ direction; (c) the view along the $-x_{3}$ direction; (d) 
the loading directions in indium for which Poisson's ratio is isotropic in the transverse plane, represented on a stereogram centred on $[001]_{V}$ showing all the directions marked from $\mathrm{A}-\mathrm{I}$ in (c). The directions have been labelled as follows: $\mathrm{A}$ is $[0.54225,0.54225,0.64181]_{V}, \mathrm{~B}$ is $[0.54225,-0.54225,0.64181]_{V}, \mathrm{C}$ is $[-0.54225,-0.54225,0.64181]_{V}, \mathrm{D}$ is $[-0.54225,0.54225,0.64181]_{V}, \mathrm{E}$ is $[0,0.93406,0.35711]_{V}, \quad \mathrm{~F}$ is $[0.93406, \quad 0,0.35711]_{V}, \quad \mathrm{G}$ is $[0,-0.93406,0.35711]_{V}, \mathrm{H}$ is $[-0.93406,0,0.35711]_{V}$ and $\mathrm{I}$ is $[001]_{V}$.

Figure 7 Representation of: (a) the anisotropy surface of zinc (point group 6/mmm); (b) the view along the $-x_{1}$ direction; (c) the view along the $-x_{3}$ direction; (d) the loading directions in zinc for which Poisson's ratio is isotropic in the transverse plane, represented on a stereogram centred on $[001]_{V}$ showing all the directions marked in (c). The directions on the circle labelled $\mathrm{A}$ are directions of the form $[\cos \theta, \sin \theta, 0.5556]_{V}$. B is $[001]_{V}$.

Figure 8 Representation of: (a) the anisotropy surface of forsterite (point group $\mathrm{mmm}$ ); (b) the view along the $-x_{2}$ direction; (c) the view along the $-x_{3}$ direction; (d) the loading directions in forsterite for which Poisson's ratio is isotropic in the transverse plane, represented on a stereogram centred on $[001]_{V}$ showing all the directions marked $\mathrm{A}-\mathrm{L}$ in (c). The directions have been labelled as follows: $\mathrm{A}$ is $[0.93515,0,0.35425]_{V}, \quad \mathrm{~B} \quad$ is $\quad[-0.93515,0,0.35425]_{V}, \quad \mathrm{C} \quad$ is $[-0.76887,0.51424,0.38000]_{V}, \quad \mathrm{D}$ is $[0.76887,0.51424,0.38000]_{V}, \quad \mathrm{E}$ is $[0.76887,-0.51424,0.38000]_{V}, \mathrm{~F}$ is $[-0.76887,-0.51424,0.38000]_{V}, \quad \mathrm{G}$ is $[-0.61839,0,0.78587]_{V}, \quad \mathrm{H}$ is $[0.61839,0,0.78587]_{V}, \quad \mathrm{I}$ is $[0.636004,-0.771685,0]_{V}, \quad \mathrm{~J} \quad$ is $[-0.636004,-0.771685,0]_{V}, \quad \mathrm{~K} \quad$ is $[-0.636004,0.771685,0]_{V}$ and $\mathrm{L}$ is $[0.636004,0.771685,0]_{V}$.

Figure 9 Representation of: (a) the anisotropy surface of magnesium silicate $\left(\mathrm{MgSiO}_{3}\right.$, point group $\overline{3}$ ); (b) the view along the $-x_{1}$ direction; (c) the view along the $-x_{3}$ direction; (d) the loading directions in magnesium silicate for which Poisson's ratio is isotropic in the transverse plane, represented on a stereogram centred on $[001]_{V}$ showing all the directions marked $\mathrm{A}-\mathrm{J}$ in (c). The directions have been labelled as follows: $\mathrm{A}$ is $[0.141798,-0.572823,0.807320]_{V}, \quad \mathrm{~B}$ is $[-0.566978,0.163611,0.807320]_{V}, \mathrm{C}$ is $[0.42518,0.409212,0.80732]_{V}, \mathrm{D}$ is $[0.638133,0.614167,0.464309]_{V}, \mathrm{E}$ is $[0.212818,-0.859723,0.464309]_{V}, \mathrm{~F}$ is $[-0.850951,0.245556,0.464309]_{V}, \mathrm{G}$ is $[-0.224692,0.907694,0.78587]_{V}, \mathrm{H}$ is $[0.898432,-0.259258,0.78587]_{V}$, I is $[-0.67374,-0.648436,0.78587]_{V}$ and $\mathrm{J}$ is $[001]_{V}$.

Figure 10 Representation of: (a) the anisotropy surface of $\alpha$-quartz (point group 32); (b) the view along the $-x_{1}$ direction; (c) the view along the $-x_{3}$ direction; (d) the loading directions in $\alpha$-quartz for which Poisson's ratio is isotropic in the transverse plane, represented on a stereogram centred on $[001]_{V}$ showing all the directions marked A-D in (c). The directions have been labelled as follows: $\mathrm{A}$ is $[-0.702479,-0.405576,0.584835]_{V}, \mathrm{~B}$ is $[0,0.811153,0.584835]_{V}, \mathrm{C}$ is $[0.702479,-0.405576,0.584835]_{V}$ and $\mathrm{D}$ is $[001]_{V}$.

Figure 11 Representation of: (a) the anisotropy surface of bismuth vanadate (point group $2 / m$ ); (b) the view along the $-x_{2}$ direction; (c) the view along the $-x_{3}$ direction; (d) the loading directions in bismuth vanadate for which Poisson's ratio is 
isotropic in the transverse plane, represented on a stereogram centred on $[001]_{V}$ showing all the directions marked $\mathrm{A}-\mathrm{F}$ in (c). The directions have been labelled as follows: $\mathrm{A}$ is $[-0.91357,0,0.40669]_{V}, \mathrm{~B}$ is $[-0.66819,0,0.74399]_{V}, \mathrm{C}$ is $[0.21772,0,0.97601]_{V}, \quad \mathrm{D} \quad$ is $[0.65434,0,0.7562]_{V}, \quad \mathrm{E}$ is $[-0.12352,-0.76776,0.62872]_{V}$ and $\mathrm{F}$ is $[-0.12352,0.76776,0.62872]_{V}$.

Figure 12 Representation of: (a) the anisotropy surface of copper sulphate pentahydrate (point group $\overline{1}$ ); (b) the view along the $-x_{1}$ direction; (c) the view along the $-x_{3}$ direction; (d) the loading directions in copper sulphate for which Poisson's ratio is isotropic in the transverse plane, represented on a stereogram centred on $[001]_{V}$ showing all the directions marked $\mathrm{A}-\mathrm{F}$ in (c). The directions have been labelled as follows: $\mathrm{A}$ is $[0.985143,-0.059928,0.160943]_{V}, \quad \mathrm{~B}$ is $[0.38437,-0.838089,0.387125]_{V}, \mathrm{C}$ is $[-0.31984,-0.716731,0.619677]_{V}, \mathrm{D}$ is $[-0.865897,0.154471,0.475775]_{V}, \mathrm{E}$ is $[-0.126077,0.919541,0.372222]_{V}$ and $\mathrm{F}$ is $[-0.865897,0.154471,0.787382]_{V}$. 


\section{List of Tables}

Table 1 Compliance coefficients (in $\left.(\mathrm{TPa})^{-1}\right)$ of the representative cubic single crystals copper and tungsten and the representative tetragonal single crystals calcium molybdate and indium.

Table 2 Compliance coefficients (in $(\mathrm{TPa})^{-1}$ ) of zinc, forsterite, $\alpha$-quartz and ilmenite representing hexagonal, orthorhombic and trigonal single crystals.

Table 3 Compliance coefficients (in $(\mathrm{TPa})^{-1}$ ) of single crystals of bismuth vanadate (monoclinic) and copper sulphate pentahydrate (triclinic).

Table 4 Loading directions for which Poisson's ratio is isotropic in the transverse plane for materials with cubic symmetry

Table 5 Loading directions for which Poisson's ratio is isotropic in the transverse plane for calcium molybdate $(4 / m)$

Table 6 Loading directions for which Poisson's ratio is isotropic in the transverse plane for indium (Point group: 4/mmm)

Table 7 Definition of the parameters $a, b$ and $c$ used to simplify the solutions for orthorhombic materials

Table 8 Loading directions for which Poisson's ratio is isotropic in the transverse plane for forsterite (Point group $\mathrm{mmm}$ )

Table 9 Loading directions for which Poisson's ratio is isotropic in the transverse plane for the ilmenite phase of magnesium silicate $\left(\mathrm{MgSiO}_{3}\right.$, point group $\left.\overline{3}\right)$

Table 10 Loading directions for which Poisson's ratio is isotropic in the transverse plane for $\alpha$-quartz (Point group 32)

Table 11 Loading directions for which Poisson's ratio is isotropic in the transverse plane for bismuth vanadate (Point group $2 / m$ )

Table 12 Loading directions for which Poisson's ratio is isotropic in the transverse plane for copper sulphate pentahydrate (Point group $\overline{1}$ ) 


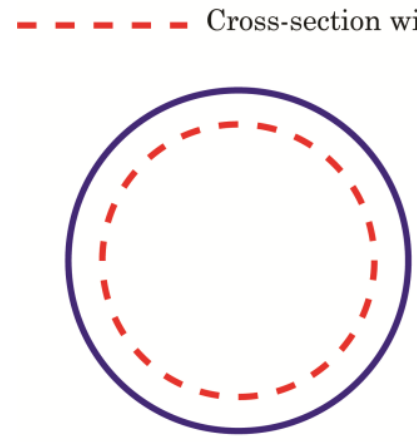

(a)

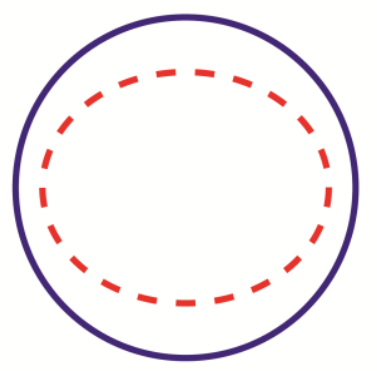

(b)

Figure 1 Schematics of elastic deformation of the cross-sections of two cylindrical specimens when loaded along their axes. (a) a circular cross-section remains circular only if the Poisson's ratio is isotropic in the transverse plane for the given loading direction. (b) a circular cross-section will not remain circular if the Poisson's ratio is not isotropic in the transverse plan. For clarity, the magnitudes of the changes for a real single crystal have been heavily exaggerated in these two schematics. 


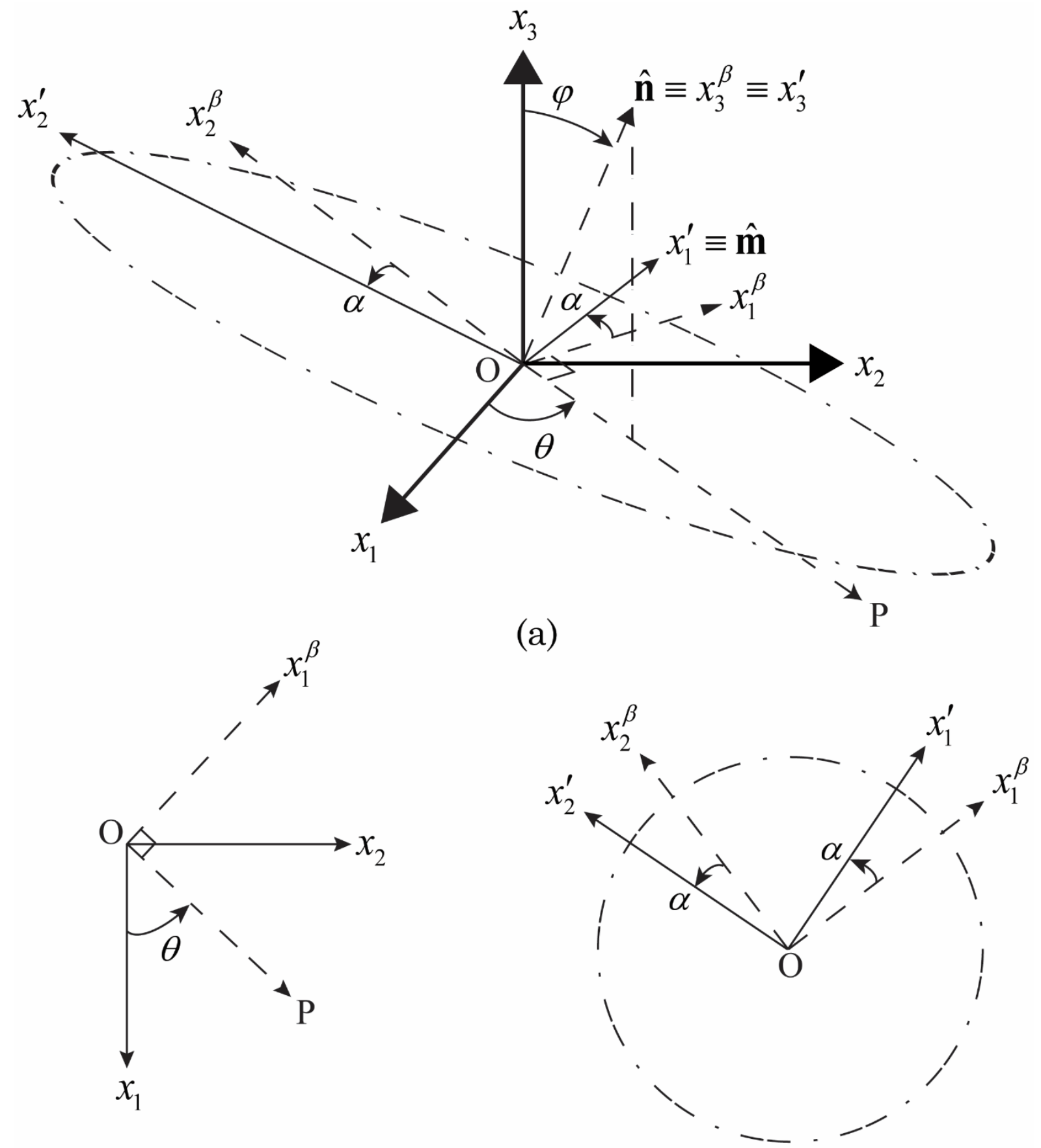

(b)

(c)

Figure 2 (a) The Voigt $\left(x_{1}, x_{2}, x_{3}\right)$ and Poisson's $\left(x_{1}^{\prime}, x_{2}^{\prime}, x_{3}^{\prime}\right)$ coordinate systems for a given loading direction ( $\hat{\mathbf{n}})$ for a rod under a uniaxial stress. The Poisson's coordinate system can be defined such that the $x_{1}^{\prime}$ axis lies along each the of the directions in the plane normal to the given loading direction by rotating the intermediate coordinate system $x_{1}^{\beta}, x_{2}^{\beta}, x_{3}^{\beta}$ by an angle $\alpha$ about the $x_{3}^{\beta}$ axis. Hence, for a given loading direction, the Poisson's ratio along each of the transverse directions can be calculated using equation (8). (b) Directions in the $x_{1}, x_{2}$ plane. OP is the projection of the loading direction $\hat{n}$ on the $x_{1}, x_{2}$ plane. (c) Directions in the plane perpendicular to the loading direction $\hat{\mathrm{n}}$ (the transverse plane). 


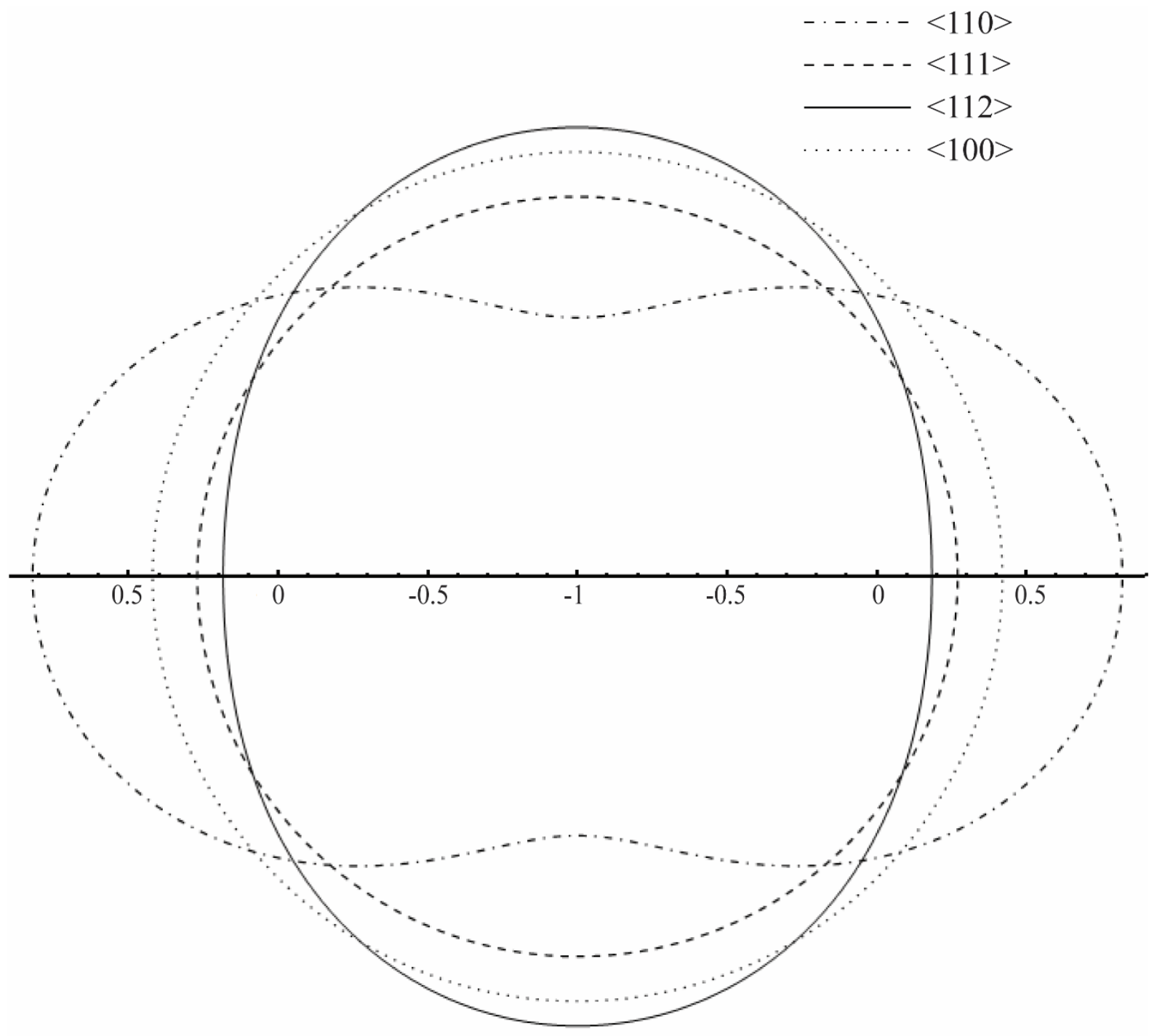

Figure 3 Variation of the Poisson's ratio of copper in the transverse plane when loaded along $\langle 110\rangle_{V},\langle 111\rangle_{V},\langle 112\rangle_{V}$ and $\langle 100\rangle_{V}$. The straight line showing the values of Poisson's ratio is along the meridional tangent of the loading direction. 


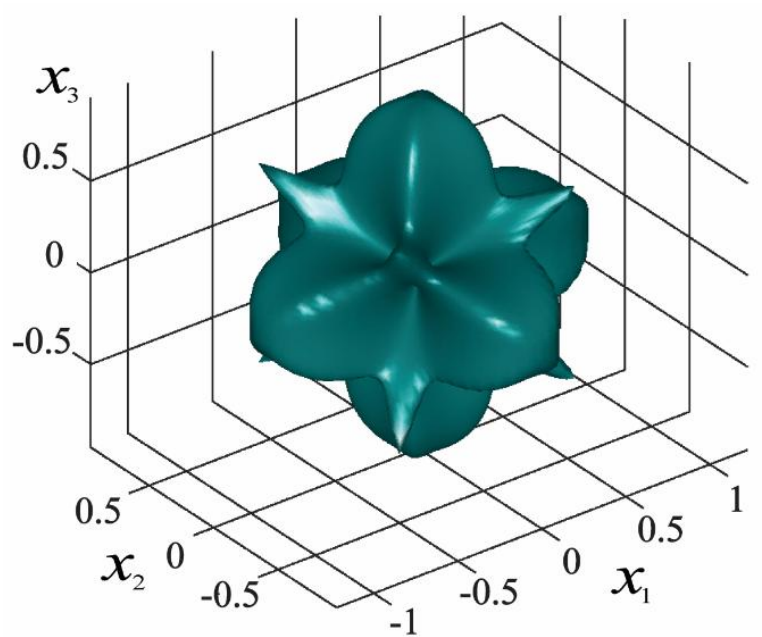

(a)

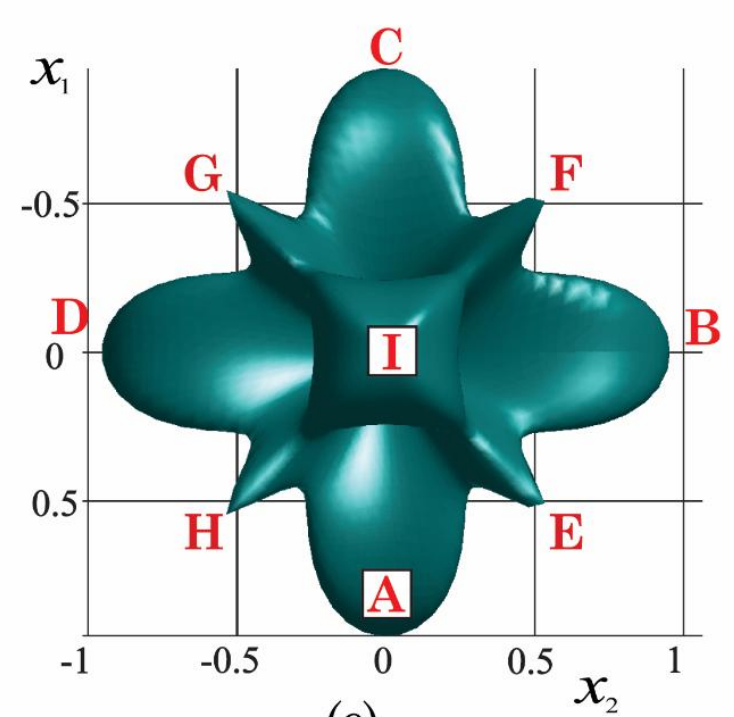

(c)

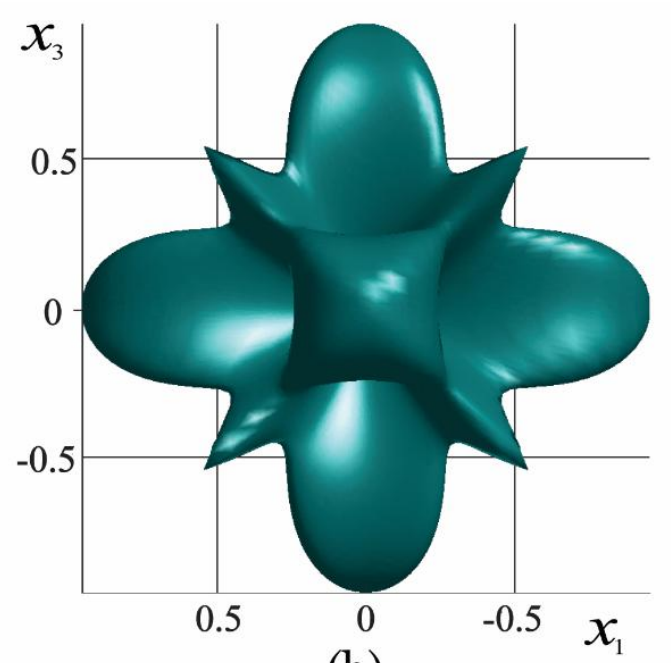

(b)

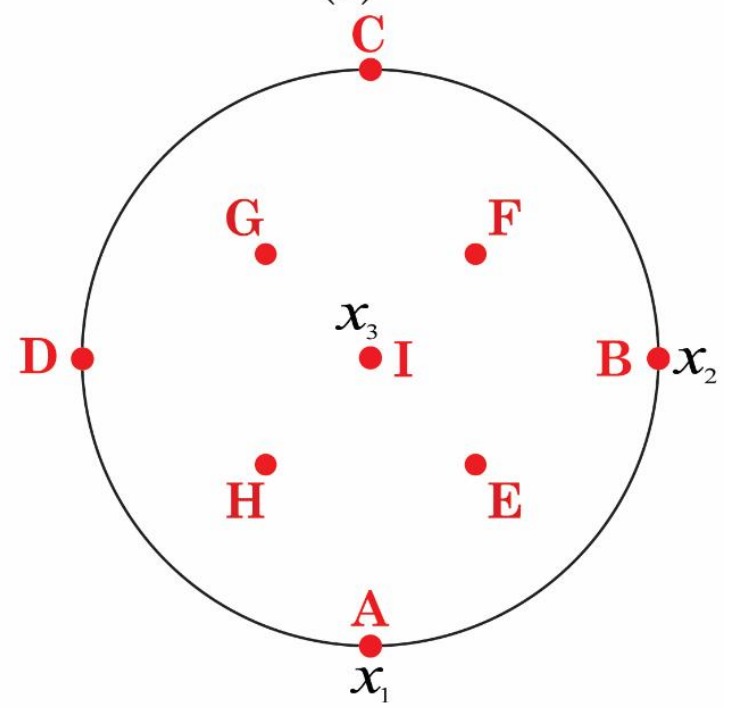

(d)

Figure 4 Representation of: (a) the anisotropy surface for copper (point group $m \overline{3} m$ ); (b) the surface viewed along the $-x_{2}$ direction; (c) the surface viewed along the $-x_{3}$ direction; (d) the loading directions in copper for which Poisson's ratio is isotropic in the transverse plane, represented on a stereogram centred at $[001]_{V}$. The directions have been labelled as follows: $\mathrm{A}$ is $[100]_{V}, \mathrm{~B}$ is $[010]_{V}, \mathrm{C}$ is $[\overline{1} 00]_{V}, \mathrm{D}$ is $[0 \overline{1} 0]_{V}, \mathrm{E}$ is $[111]_{V}, \mathrm{~F}$ is $[\overline{1} 11]_{V}, \mathrm{G}$ is $[\overline{1} \overline{1} 1]_{V}, \mathrm{H}$ is $[1 \overline{1} 1]_{V}$ and $\mathrm{I}$ is $[001]_{V}$. For clarity, only loading directions in the northern hemisphere and on the equatorial plane have been specified in the stereogram in (d) and other stereograms in this paper. 


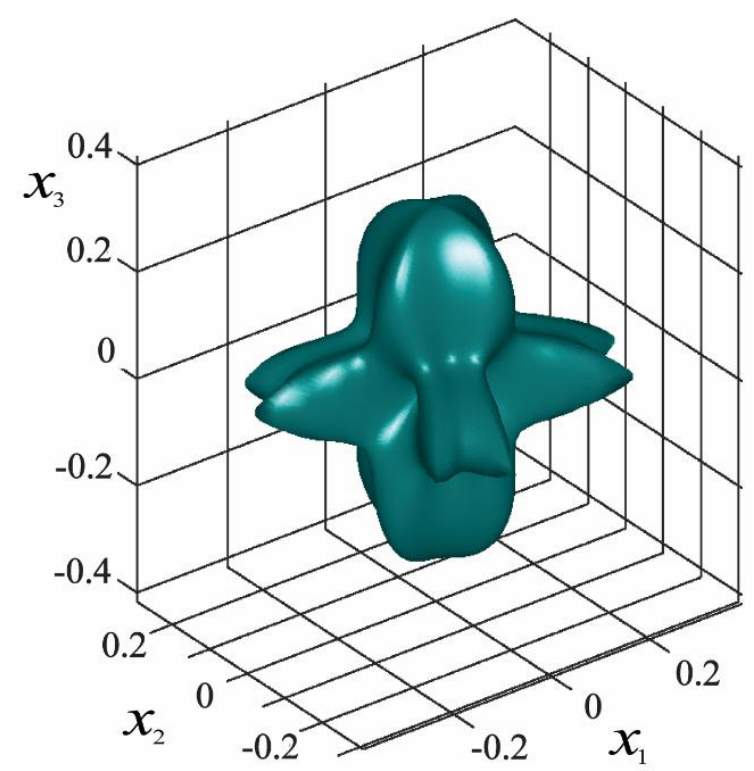

(a)

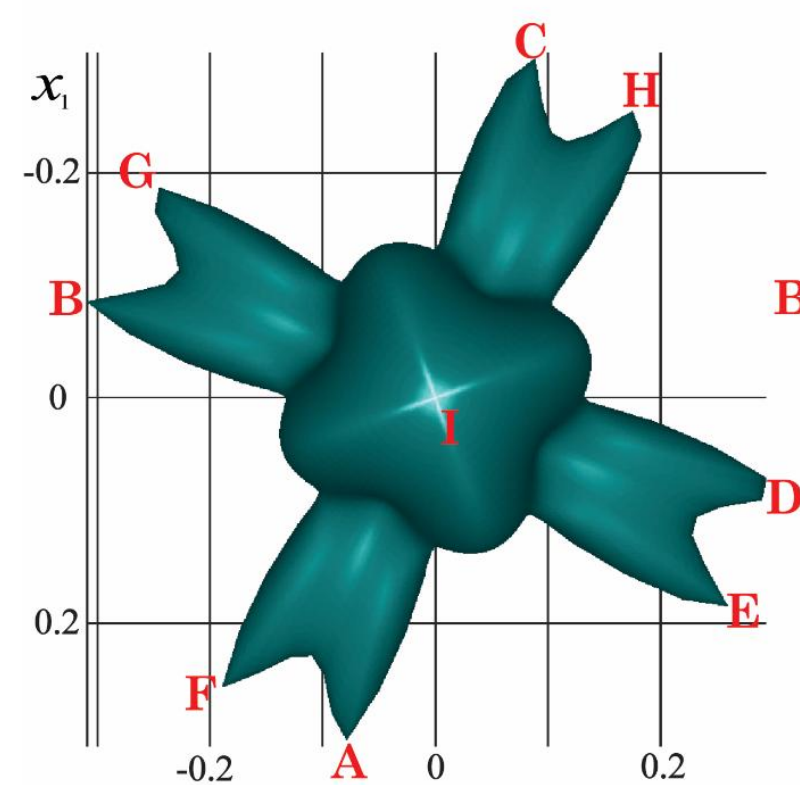

(c)

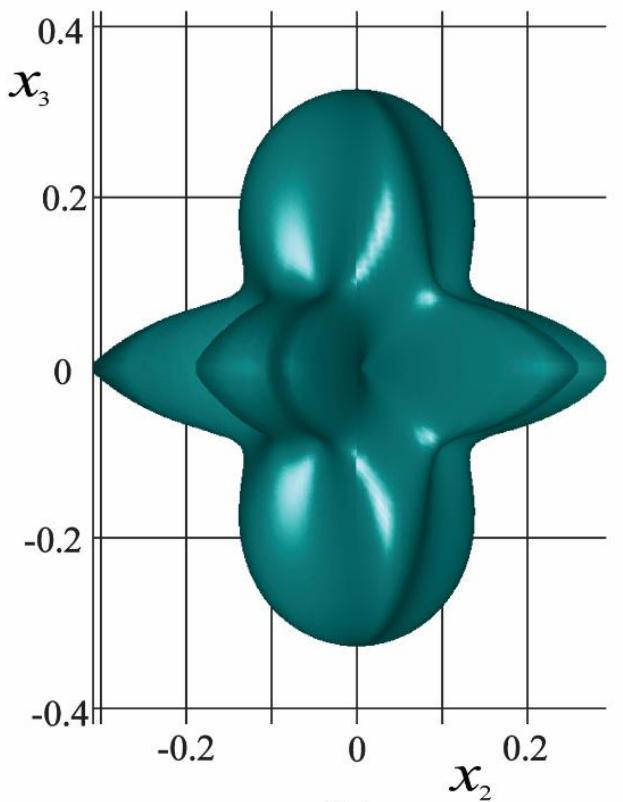

(b)

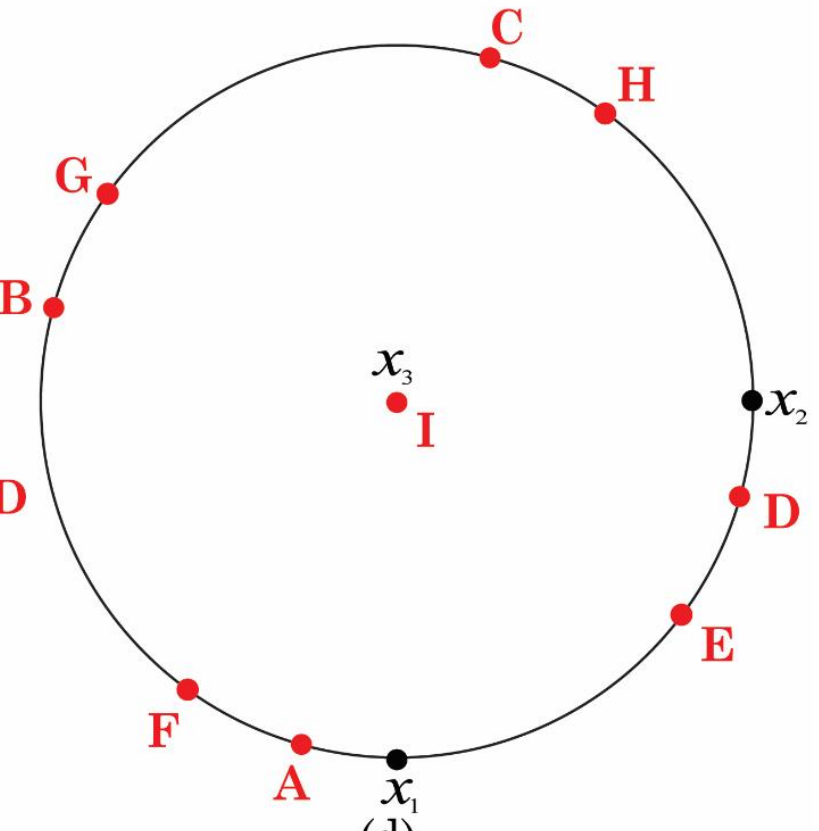

(d)

Figure 5 Representation of: (a) the anisotropy surface of calcium molybdate (point group $4 / m)$; (b) the view along the $-x_{1}$ direction; (c) the view along the $-x_{3}$ direction; (d) the loading directions in calcium molybdate for which Poisson's ratio is isotropic in the transverse plane, represented on a stereogram centred on $[001]_{V}$ showing all the directions marked from $\mathrm{A}-\mathrm{I}$ in (c). The directions have been labelled as follows: $\mathrm{A}$ is $[0.962155,-0.272503,0]_{V}, \mathrm{~B}$ is $[-0.272503$, $-0.962155,0]_{V}, \mathrm{C}$ is $[-0.962155,0.272503,0]_{V}, \mathrm{D}$ is $[0.272503,0.962155,0]_{V}$, $\mathrm{E}$ is $[0.496618,0.867969,0]_{V}, \quad \mathrm{~F}$ is $[0.867969,-0.496618,0]_{V}, \quad \mathrm{G}$ is $[-0.496618,-0.867969,0]_{V}, \mathrm{H}$ is $[-0.867969,0.496618,0]_{V}$ and $\mathrm{I}$ is $[001]_{V}$. 


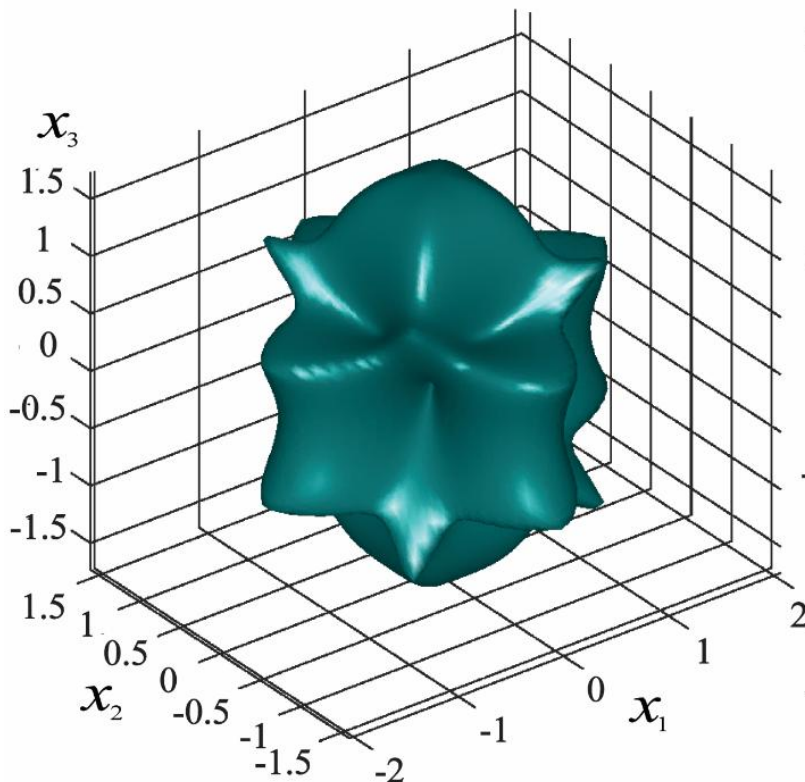

(a)

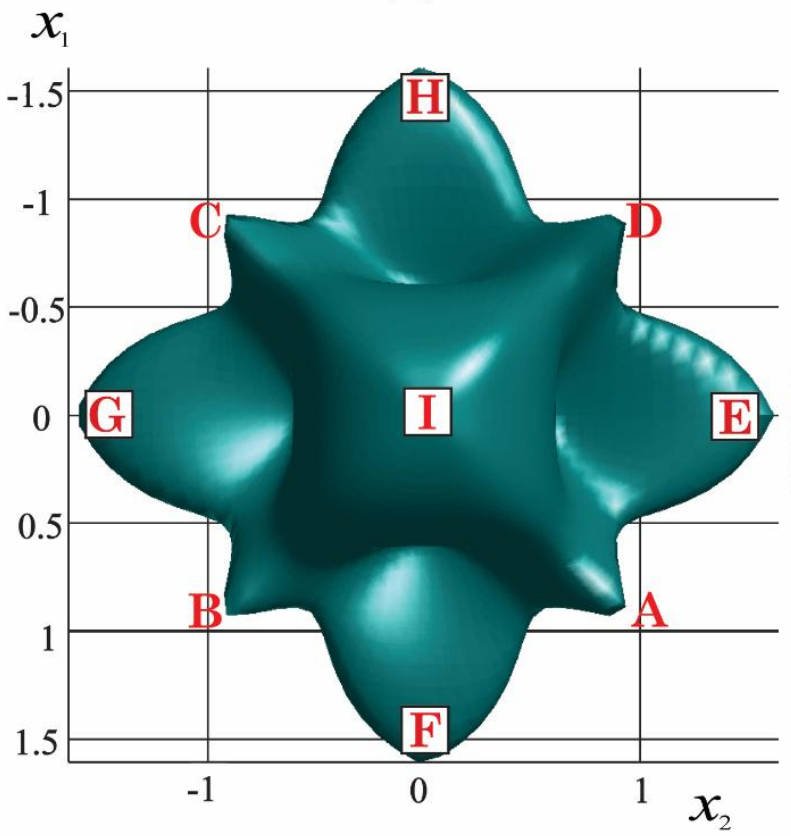

(c)

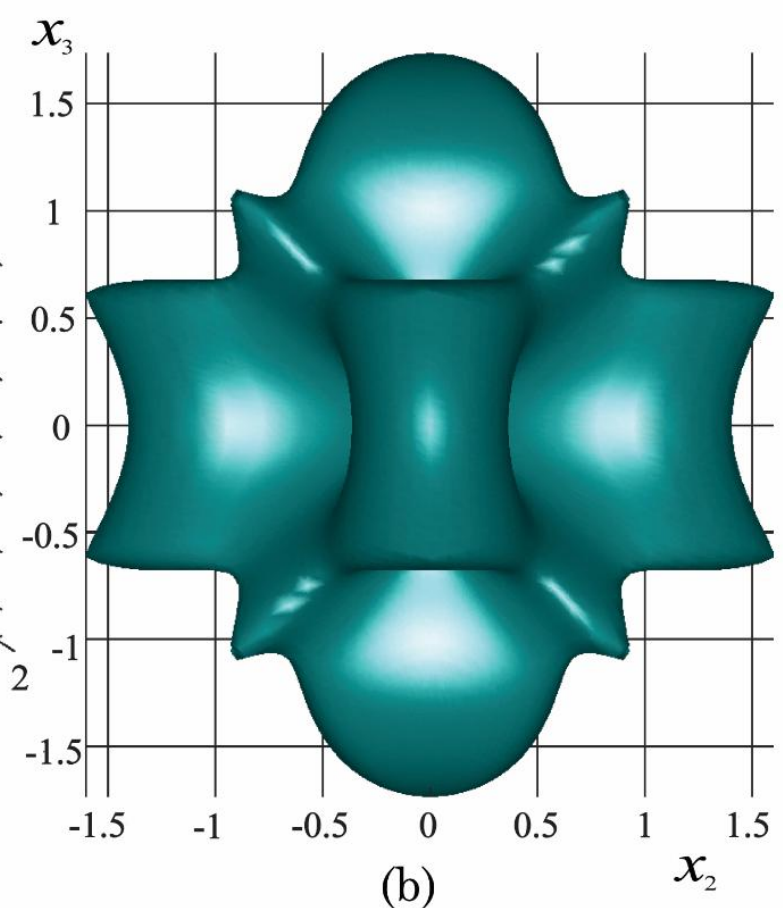

(b)

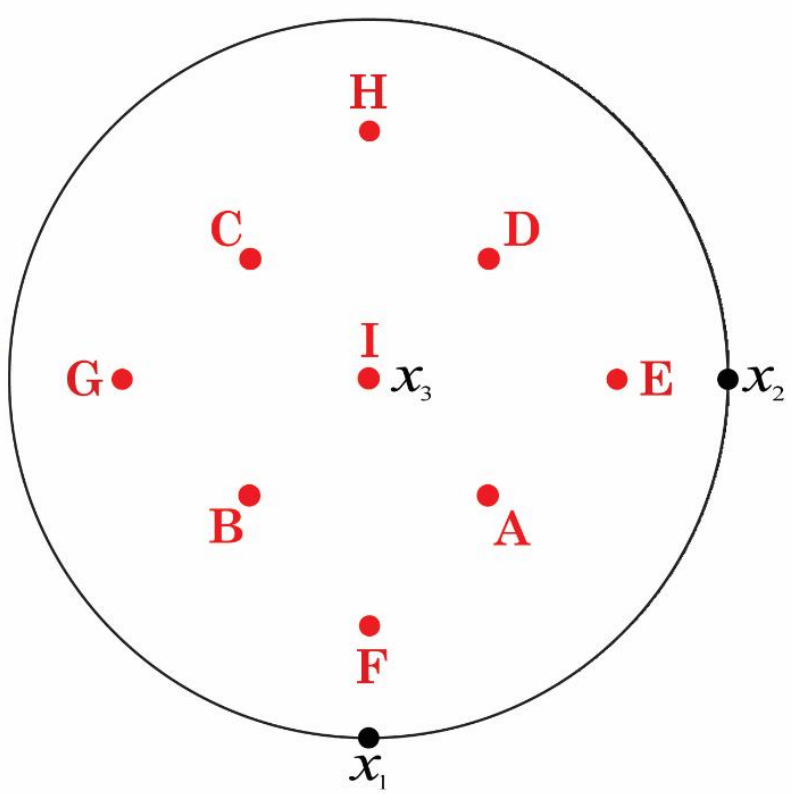

(d)

Figure 6 Representation of: (a) the anisotropy surface of indium (point group 4/mmm); (b) the view along the $-x_{1}$ direction; (c) the view along the $-x_{3}$ direction; (d) the loading directions in indium for which Poisson's ratio is isotropic in the transverse plane, represented on a stereogram centred on $[001]_{V}$ showing all the directions marked from $\mathrm{A}-\mathrm{I}$ in (c). The directions have been labelled as follows: $\mathrm{A}$ is $[0.54225,0.54225,0.64181]_{V}, \mathrm{~B}$ is $[0.54225,-0.54225,0.64181]_{V}, \mathrm{C}$ is $[-0.54225,-0.54225,0.64181]_{V}, \mathrm{D}$ is $[-0.54225,0.54225,0.64181]_{V}, \mathrm{E}$ is $[0,0.93406,0.35711]_{V}, \quad \mathrm{~F}$ is $[0.93406, \quad 0,0.35711]_{V}, \quad \mathrm{G}$ is $[0,-0.93406,0.35711]_{V}, \mathrm{H}$ is $[-0.93406,0,0.35711]_{V}$ and $\mathrm{I}$ is $[001]_{V}$. 


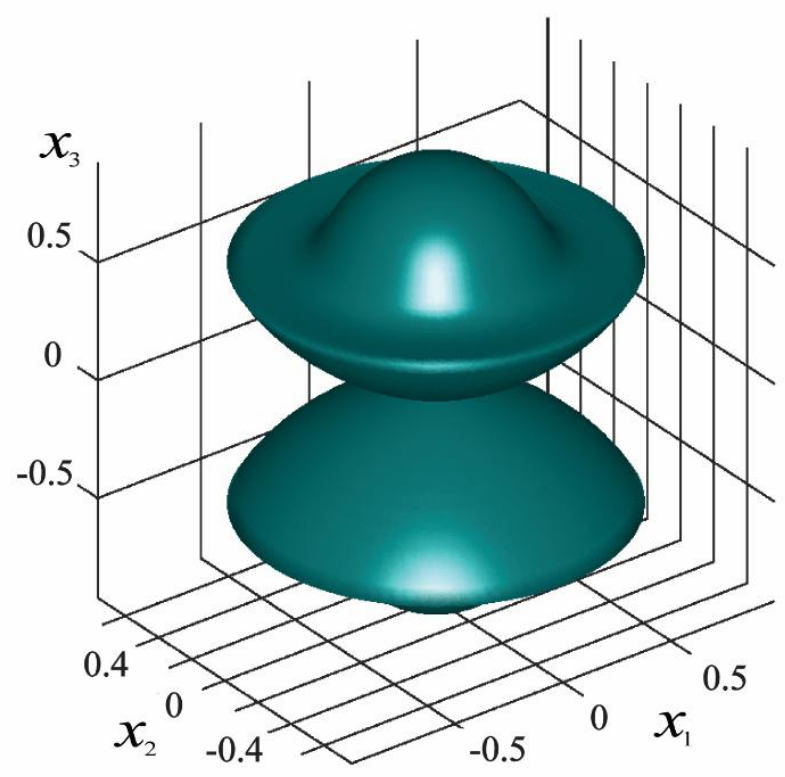

(a)

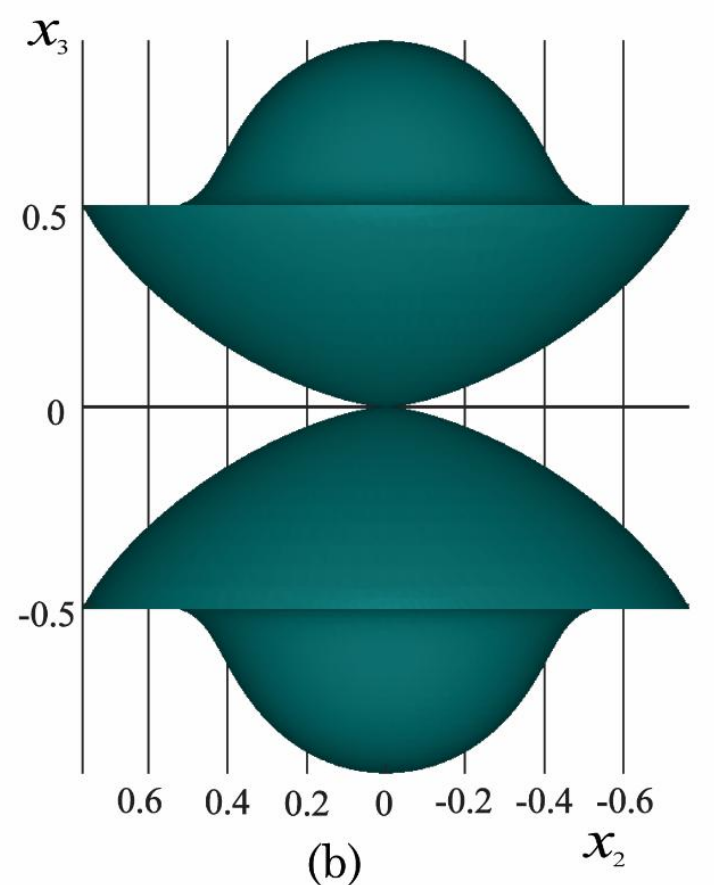

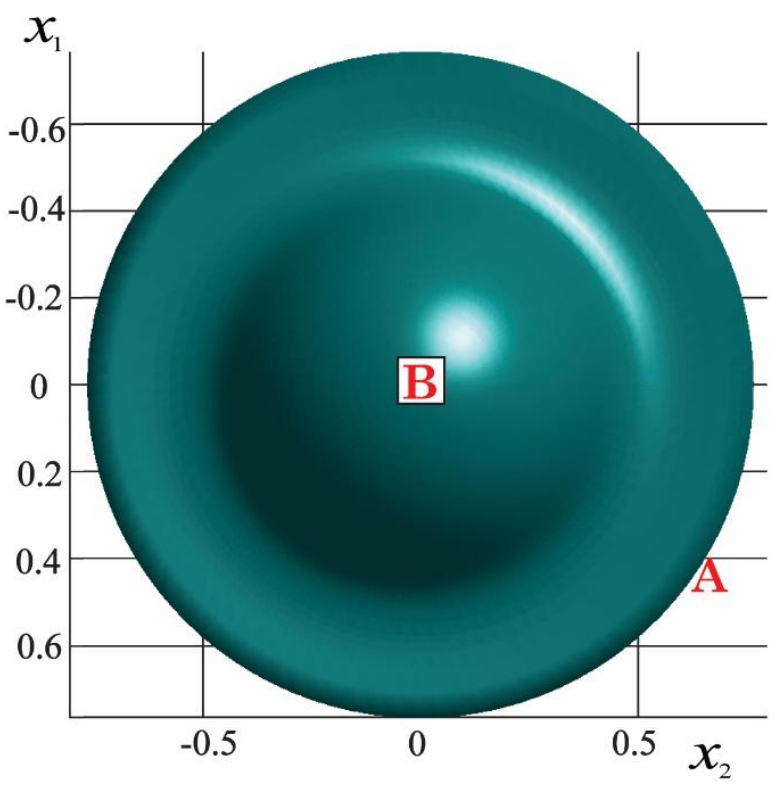

(c)

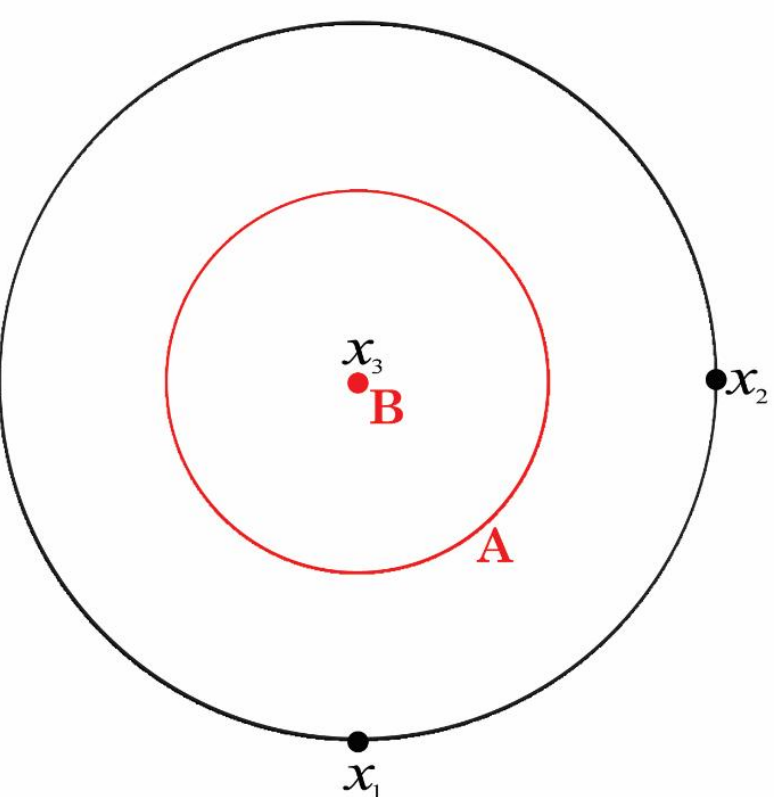

(d)

Figure 7 Representation of: (a) the anisotropy surface of zinc (point group 6/mmm); (b) the view along the $-x_{1}$ direction; (c) the view along the $-x_{3}$ direction; (d) the loading directions in zinc for which Poisson's ratio is isotropic in the transverse plane, represented on a stereogram centred on $[001]_{V}$ showing all the directions marked in (c). The directions on the circle labelled $\mathrm{A}$ are directions of the form $[\cos \theta, \sin \theta, 0.5556]_{V}$. B is $[001]_{V}$. 


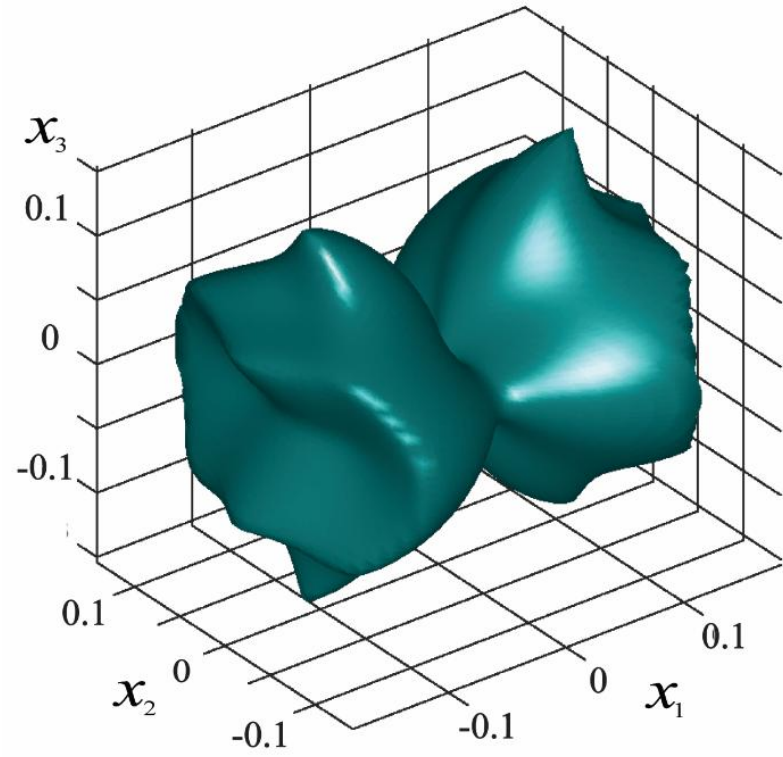

(a)

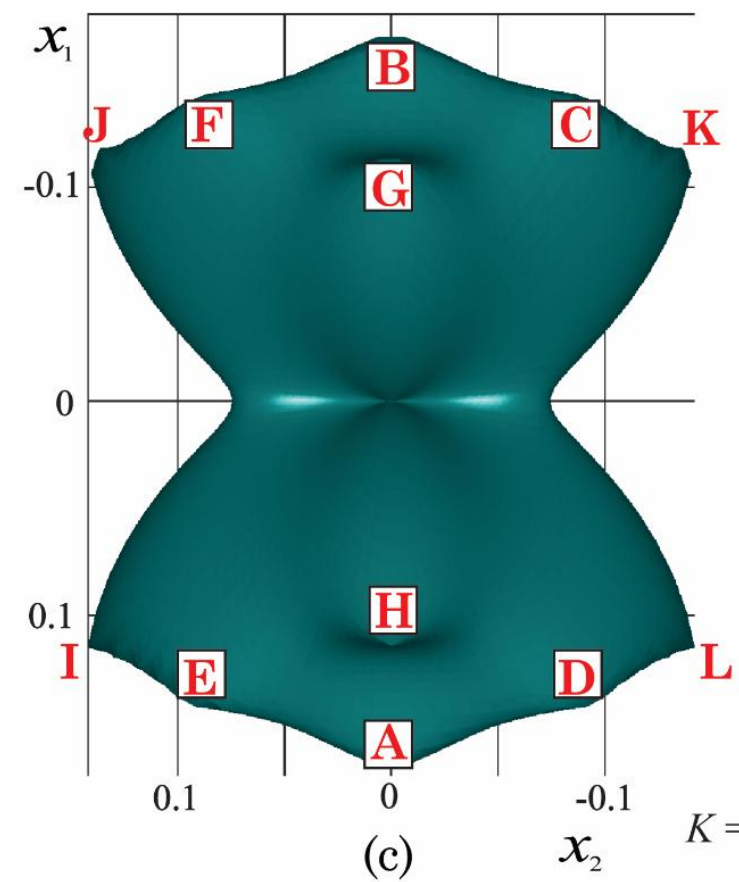

(c)

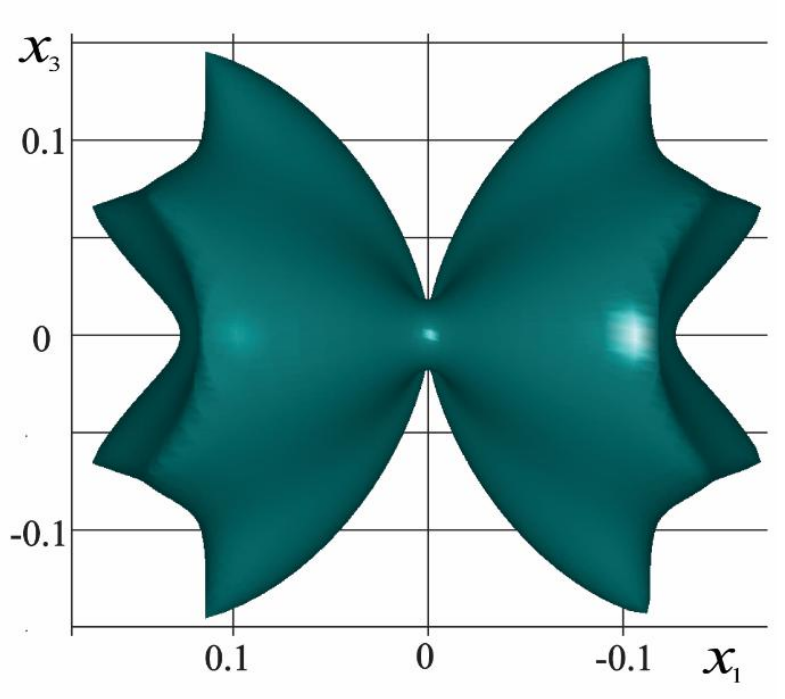

(b)

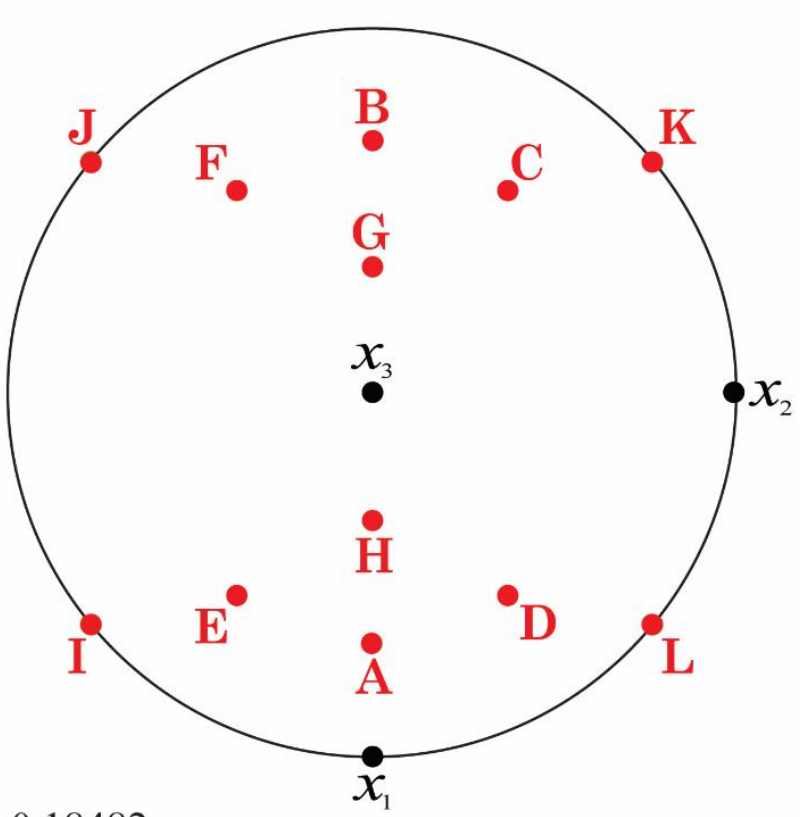

(d)

Figure 8 Representation of: (a) the anisotropy surface of forsterite (point group $\mathrm{mmm}$ ); (b) the view along the $-x_{2}$ direction; (c) the view along the $-x_{3}$ direction; (d) the loading directions in forsterite for which Poisson's ratio is isotropic in the transverse plane, represented on a stereogram centred on $[001]_{V}$ showing all the directions marked $\mathrm{A}-\mathrm{L}$ in (c). The directions have been labelled as follows: $\mathrm{A}$ is $[0.93515,0,0.35425]_{V}, \quad \mathrm{~B} \quad$ is $[-0.93515,0,0.35425]_{V}, \quad \mathrm{C} \quad$ is $[-0.76887,0.51424,0.38000]_{V}, \quad \mathrm{D}$ is $[0.76887,0.51424,0.38000]_{V}, \quad \mathrm{E}$ is $[0.76887,-0.51424,0.38000]_{V}, \mathrm{~F}$ is $[-0.76887,-0.51424,0.38000]_{V}, \quad \mathrm{G}$ is $[-0.61839,0,0.78587]_{V}, \quad \mathrm{H}$ is $[0.61839,0,0.78587]_{V}, \quad \mathrm{I}$ is $[0.636004,-0.771685,0]_{V}, \quad \mathrm{~J} \quad$ is $\quad[-0.636004,-0.771685,0]_{V}, \quad \mathrm{~K} \quad$ is $[-0.636004,0.771685,0]_{V}$ and $\mathrm{L}$ is $[0.636004,0.771685,0]_{V}$. 


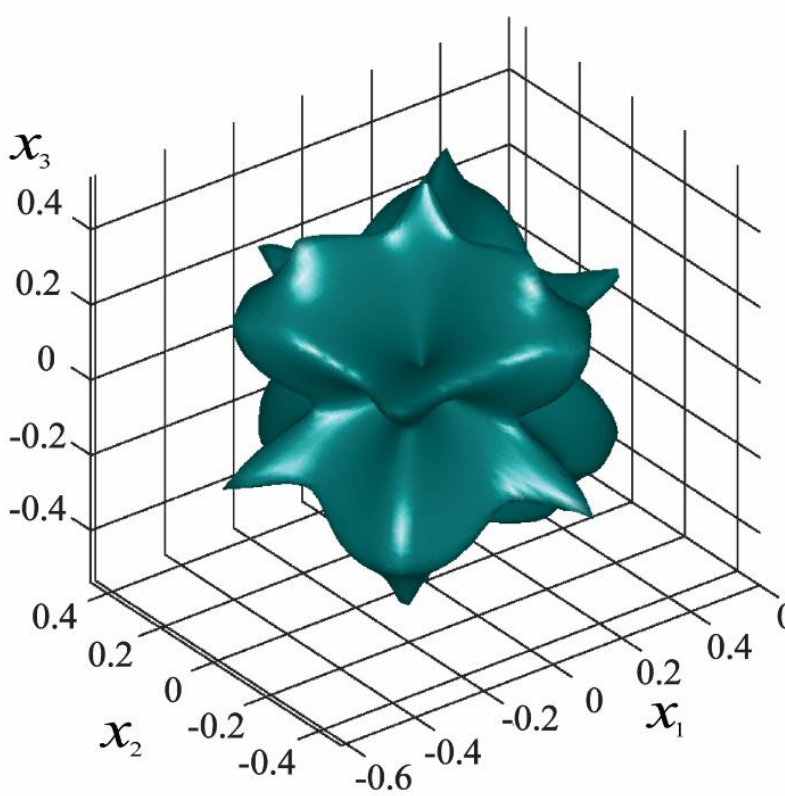

(a)

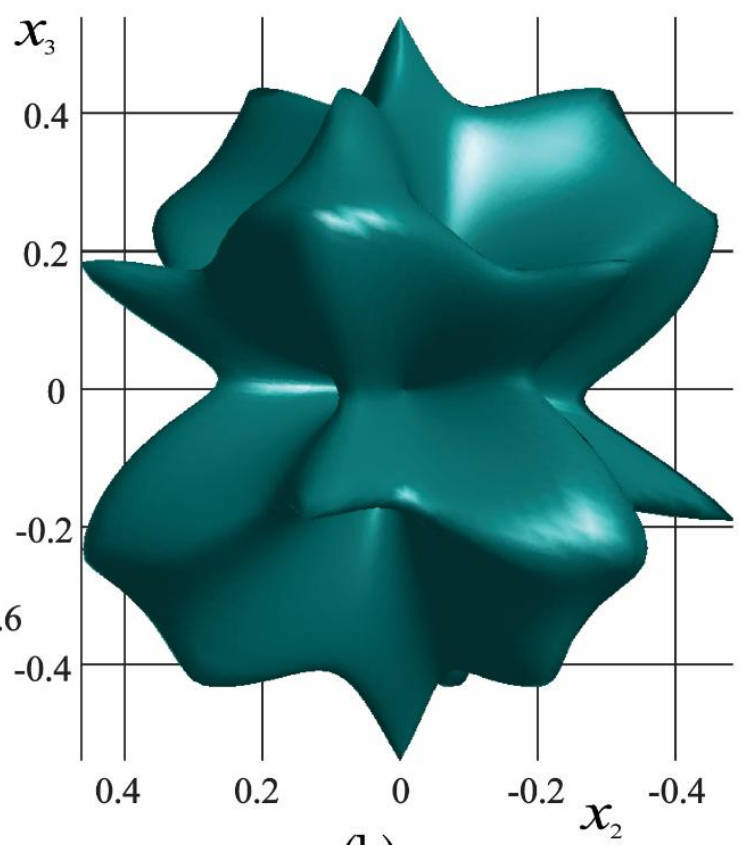

(b)

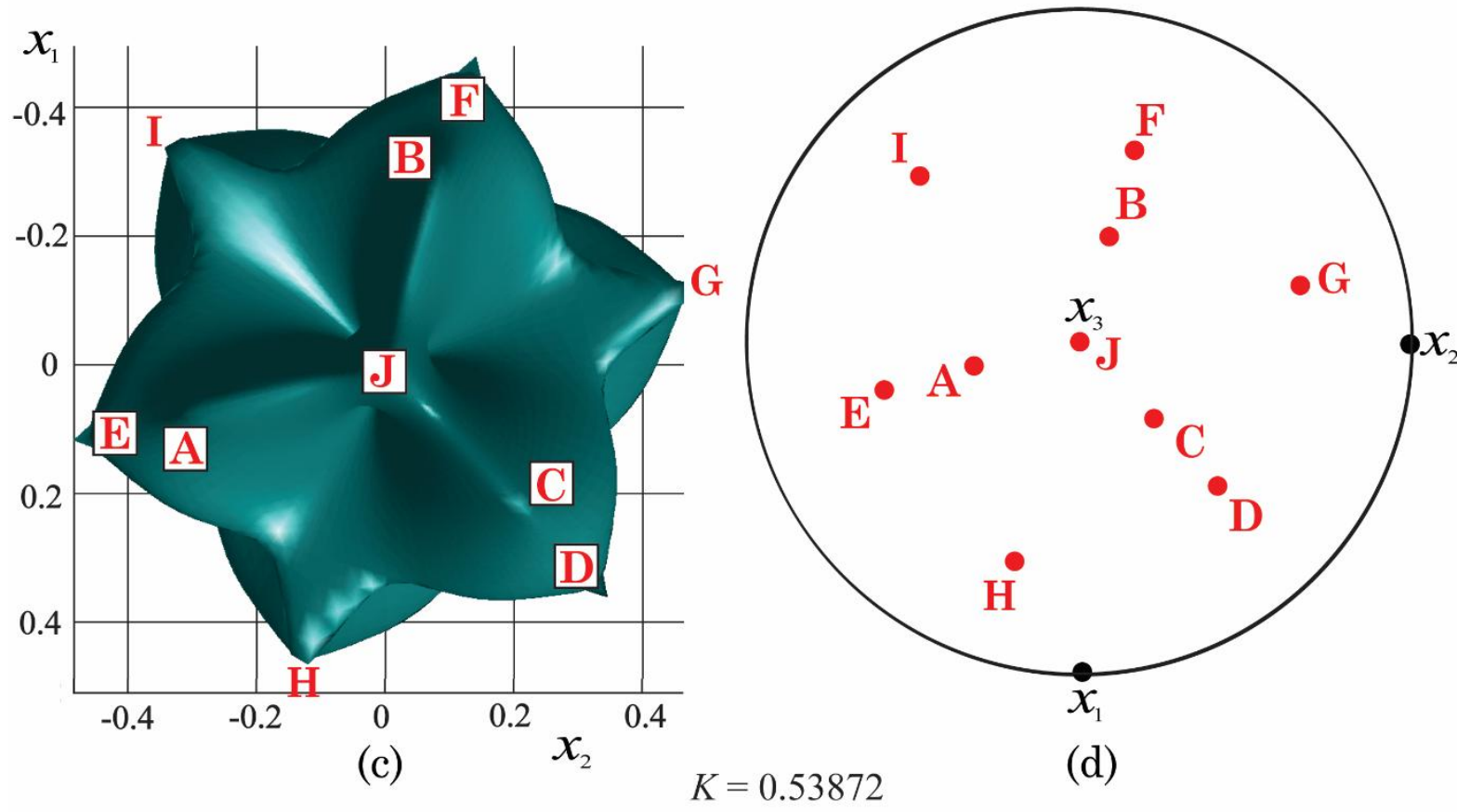

Figure 9 Representation of: (a) the anisotropy surface of magnesium silicate $\left(\mathrm{MgSiO}_{3}\right.$, point group $\overline{3}$ ); (b) the view along the $-x_{1}$ direction; (c) the view along the $-x_{3}$ direction; (d) the loading directions in magnesium silicate for which Poisson's ratio is isotropic in the transverse plane, represented on a stereogram centred on $[001]_{V}$ showing all the directions marked A-J in (c). The directions have been labelled as follows: $\mathrm{A}$ is $[0.141798,-0.572823,0.807320]_{V}, \quad \mathrm{~B}$ is $[-0.566978,0.163611,0.807320]_{V}, \mathrm{C}$ is $[0.42518,0.409212,0.80732]_{V}, \mathrm{D}$ is $[0.638133,0.614167,0.464309]_{V}, \mathrm{E}$ is $[0.212818,-0.859723,0.464309]_{V}, \mathrm{~F}$ is $[-0.850951,0.245556,0.464309]_{V}, \mathrm{G}$ is $[-0.224692,0.907694,0.78587]_{V}, \mathrm{H}$ is $[0.898432,-0.259258,0.78587]_{V}, \mathrm{I}$ is $[-0.67374,-0.648436,0.78587]_{V}$ and $\mathrm{J}$ is $[001]_{V}$. 


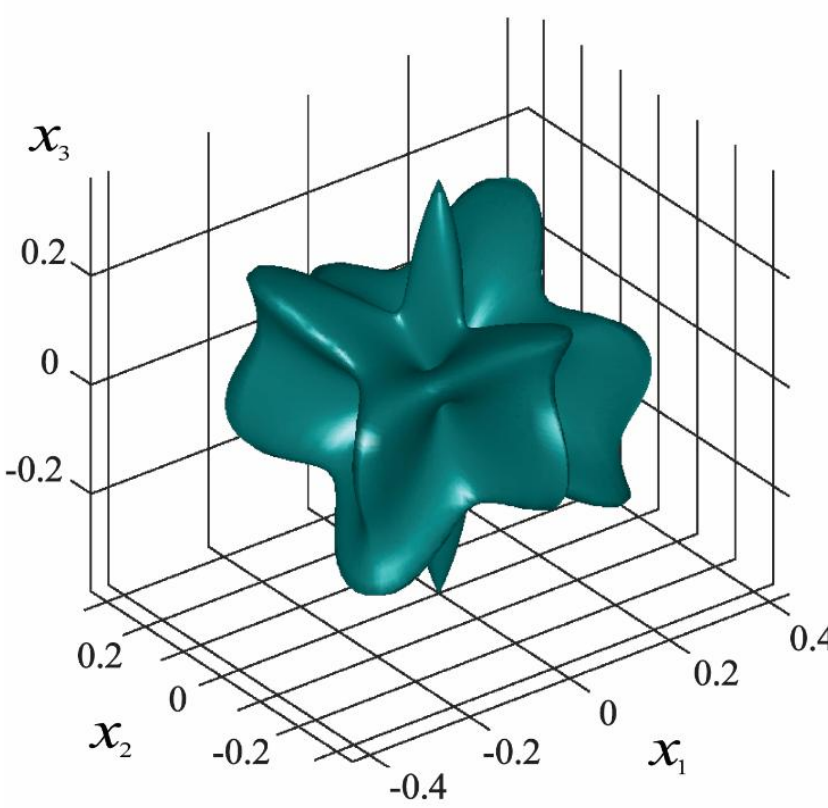

(a)

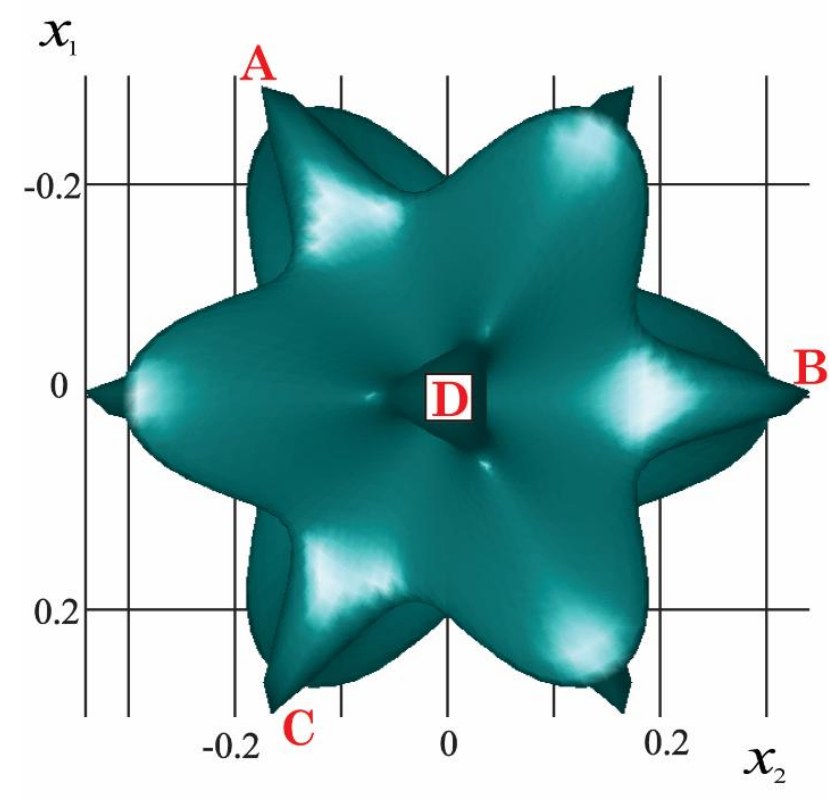

(c)

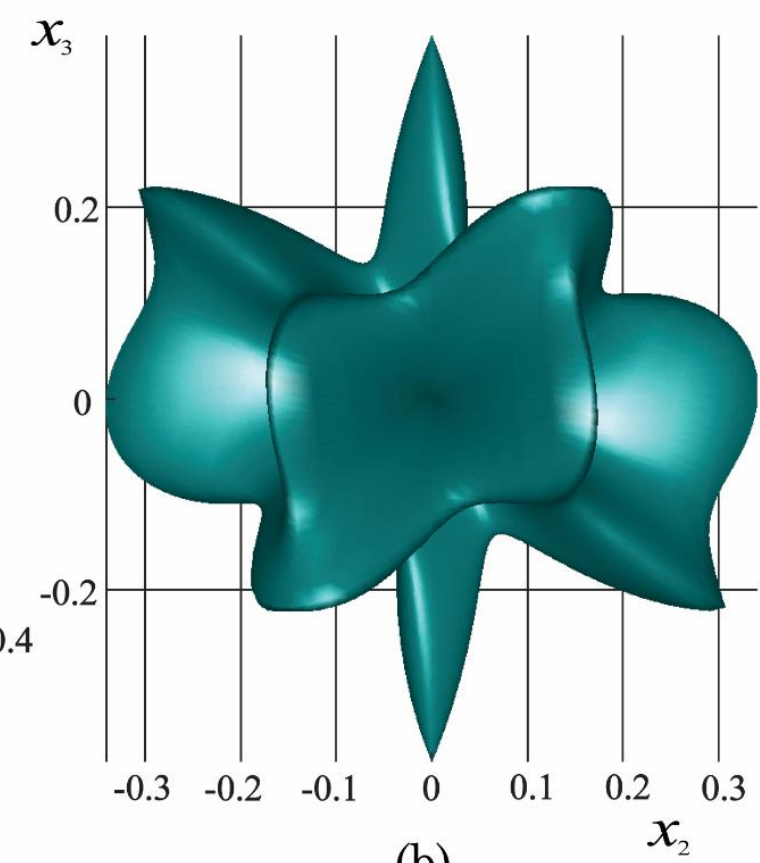

(b)

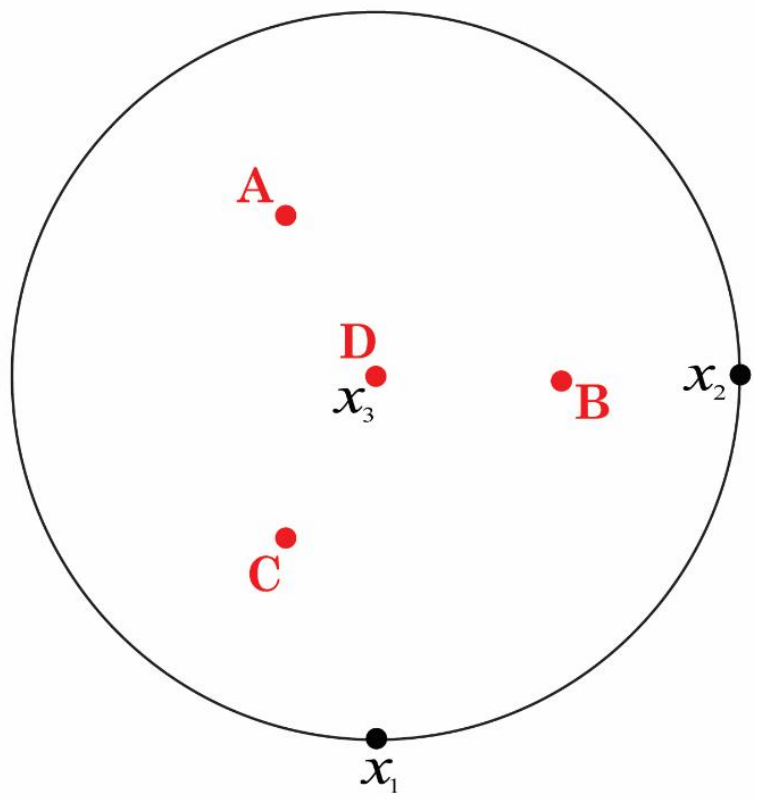

(d)

$$
K=0.37937
$$

Figure 10 Representation of: (a) the anisotropy surface of $\alpha$-quartz (point group 32); (b) the view along the $-x_{1}$ direction; (c) the view along the $-x_{3}$ direction; (d) the loading directions in $\alpha$-quartz for which Poisson's ratio is isotropic in the transverse plane, represented on a stereogram centred on $[001]_{V}$ showing all the directions marked A-D in (c). The directions have been labelled as follows: A is $[-0.702479,-0.405576,0.584835]_{V}, \mathrm{~B}$ is $[0,0.811153,0.584835]_{V}, \mathrm{C}$ is $[0.702479,-0.405576,0.584835]_{V}$ and $\mathrm{D}$ is $[001]_{V}$. 


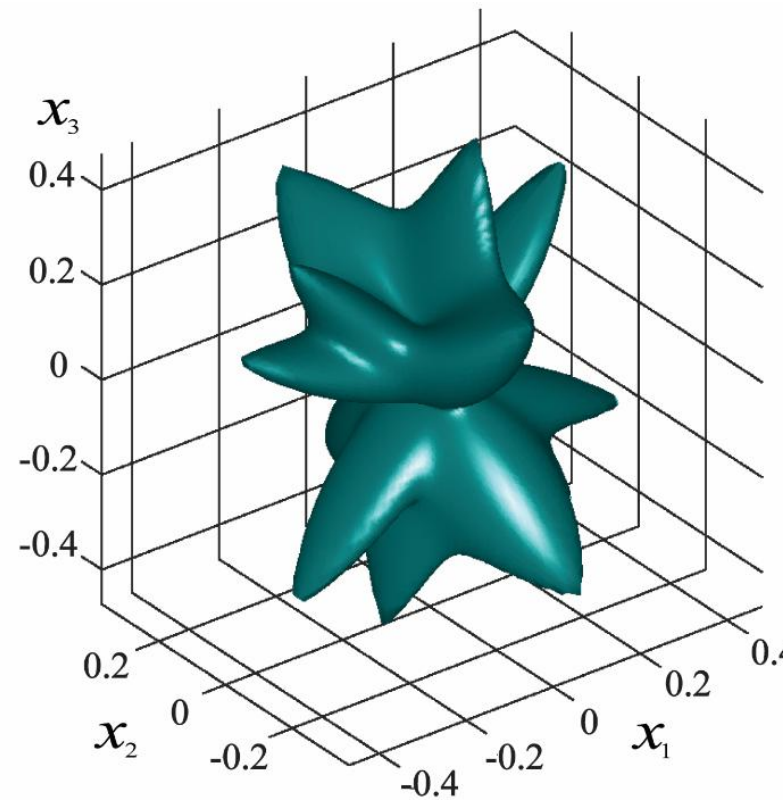

(a)

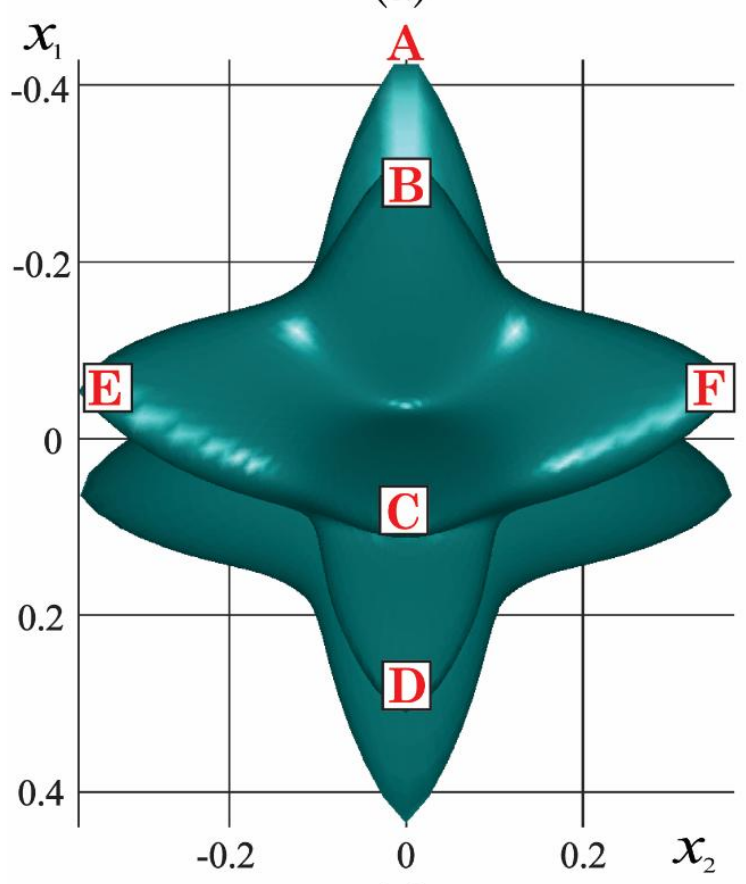

(c)

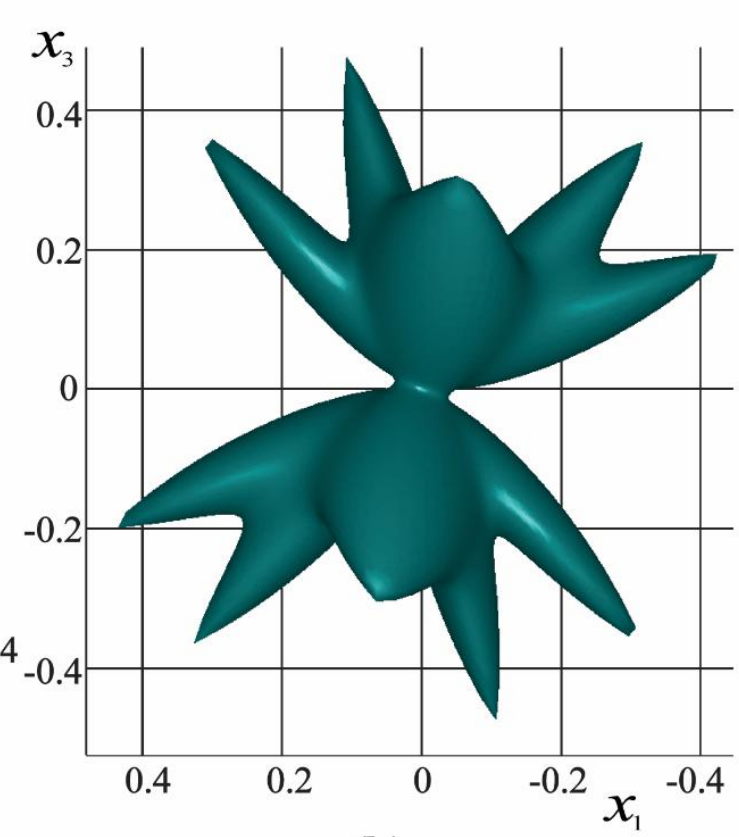

(b)

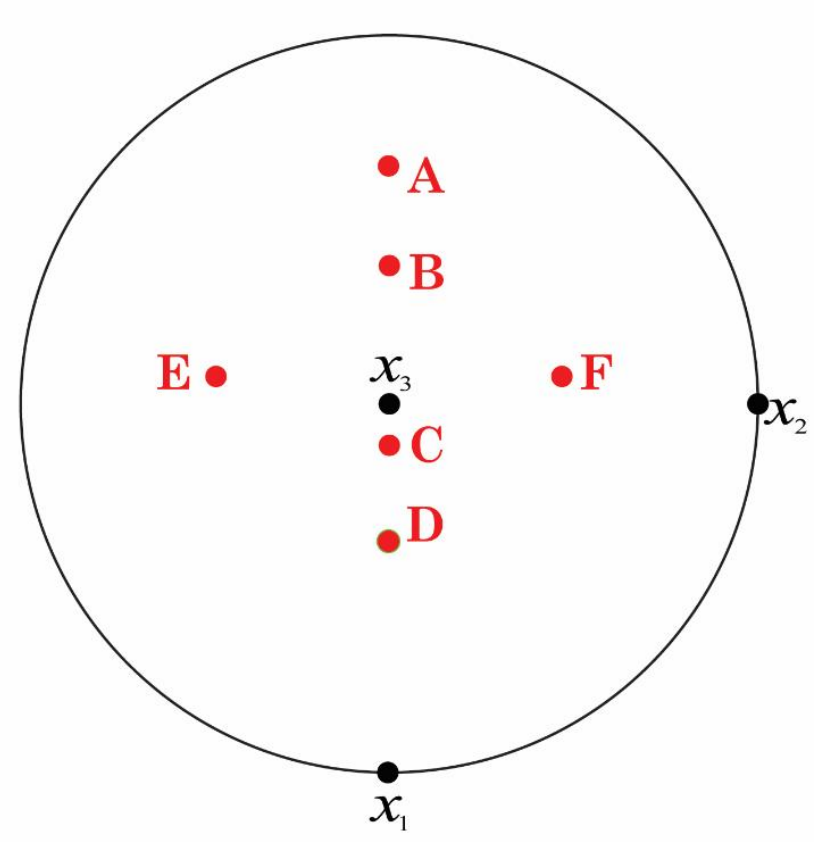

(d)

Figure 11 Representation of: (a) the anisotropy surface of bismuth vanadate (point group $2 / m$ ); (b) the view along the $-x_{2}$ direction; (c) the view along the $-x_{3}$ direction; (d) the loading directions in bismuth vanadate for which Poisson's ratio is isotropic in the transverse plane, represented on a stereogram centred on [001] $]_{V}$ showing all the directions marked $\mathrm{A}-\mathrm{F}$ in (c). The directions have been labelled as follows: $\mathrm{A}$ is $[-0.91357,0,0.40669]_{V}, \mathrm{~B}$ is $[-0.66819,0,0.74399]_{V}, \mathrm{C}$ is $[0.21772,0,0.97601]_{V}, \quad \mathrm{D} \quad$ is $[0.65434,0,0.7562]_{V}, \quad \mathrm{E}$ is $[-0.12352,-0.76776,0.62872]_{V}$ and $\mathrm{F}$ is $[-0.12352,0.76776,0.62872]_{V}$. 


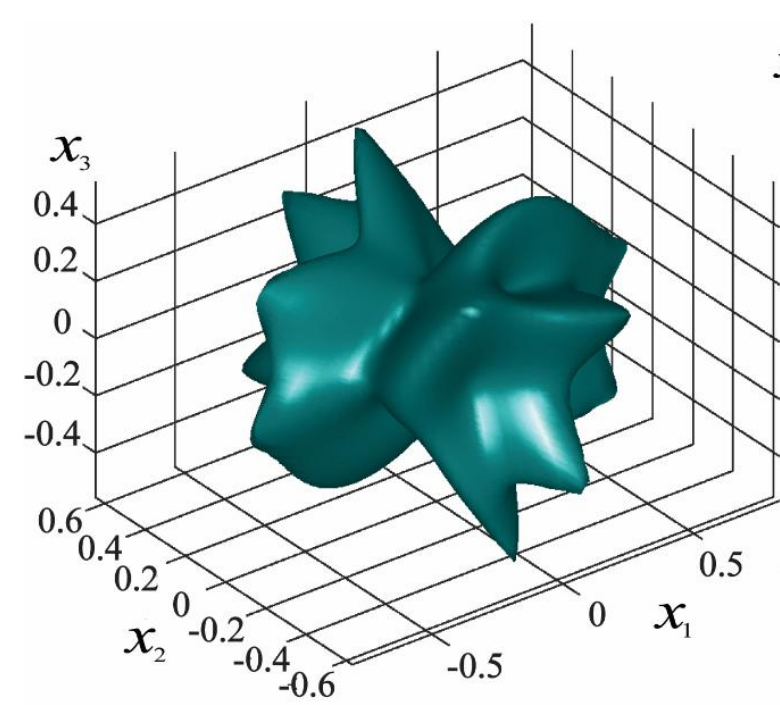

(a)

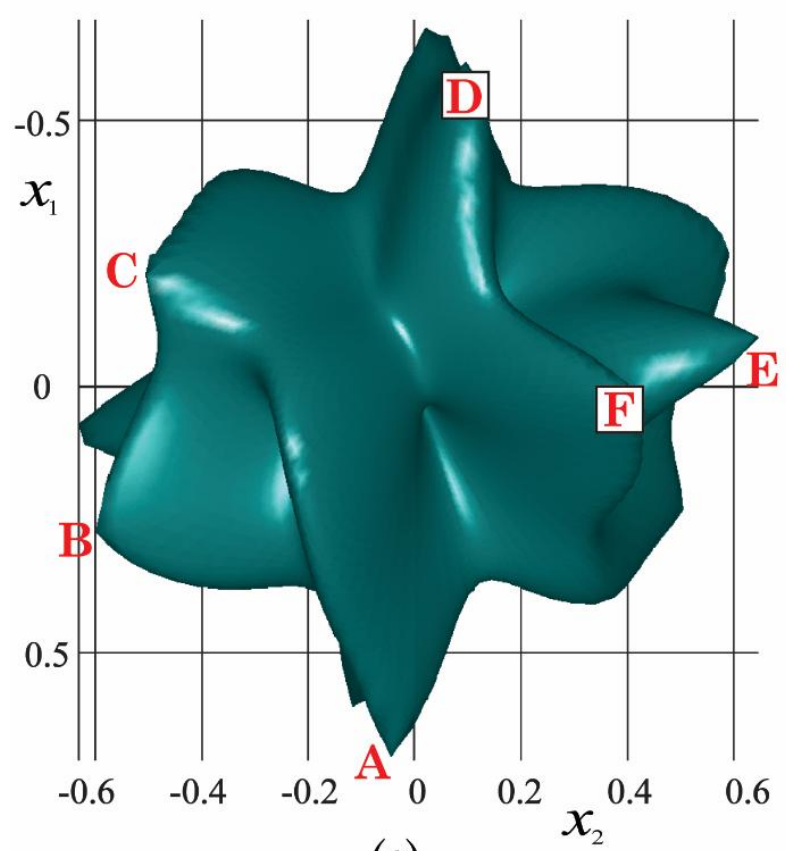

(c)

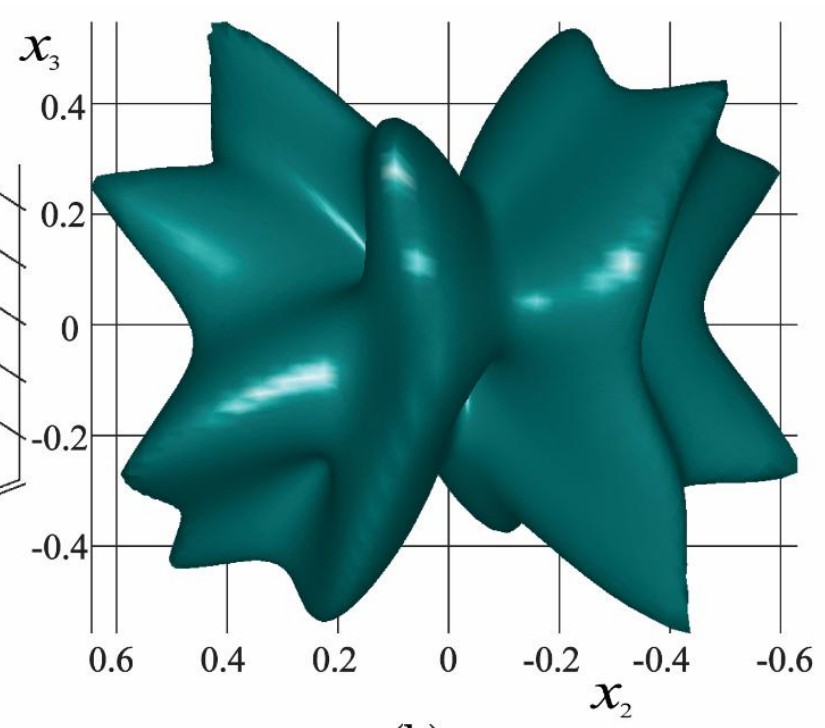

(b)

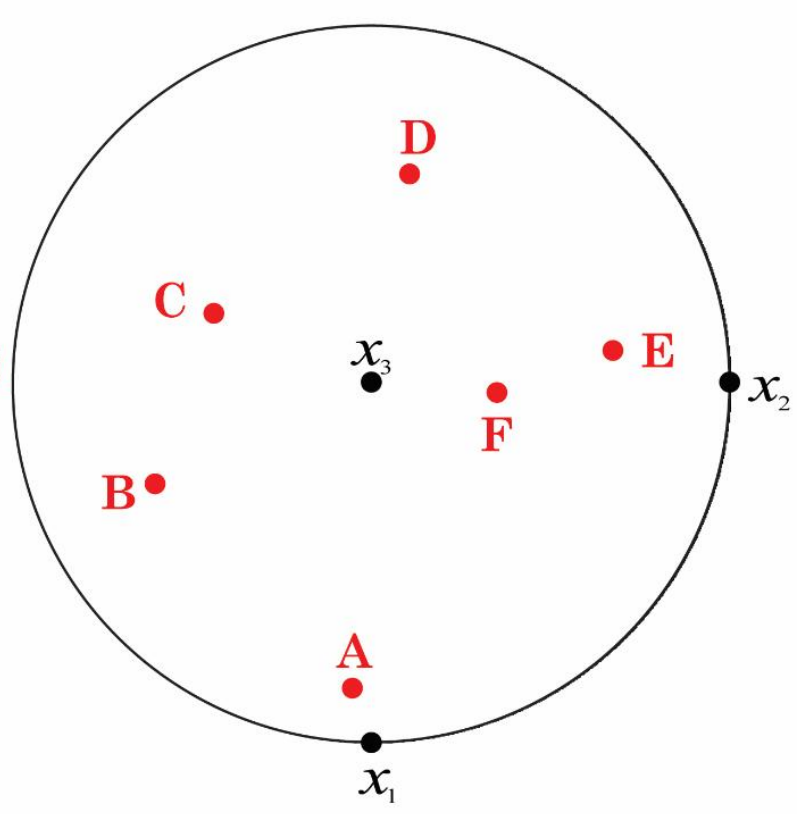

(d)

Figure 12 Representation of: (a) the anisotropy surface of copper sulphate pentahydrate (point group $\overline{1}$ ); (b) the view along the $-x_{1}$ direction; (c) the view along the $-x_{3}$ direction; (d) the loading directions in copper sulphate for which Poisson's ratio is isotropic in the transverse plane, represented on a stereogram centred on $[001]_{V}$ showing all the directions marked $\mathrm{A}-\mathrm{F}$ in $(\mathrm{c})$. The directions have been labelled as follows: $\mathrm{A}$ is $[0.985143,-0.059928,0.160943]_{V}, \quad \mathrm{~B}$ is $[0.38437,-0.838089,0.387125]_{V}, \mathrm{C}$ is $[-0.31984,-0.716731,0.619677]_{V}, \mathrm{D}$ is $[-0.865897,0.154471,0.475775]_{V}, \mathrm{E}$ is $[-0.126077,0.919541,0.372222]_{V}$ and $\mathrm{F}$ is $[-0.865897,0.154471,0.787382]_{V}$. 


\section{Tables}

Table 1 Compliance coefficients (in $(\mathrm{TPa})^{-1}$ ) of the representative cubic single crystals copper and tungsten and the representative tetragonal single crystals calcium molybdate and indium

\begin{tabular}{ccccc}
\hline Crystal & Copper & Tungsten & $\begin{array}{c}\text { Calcium } \\
\text { molybdate }\end{array}$ & Indium \\
\hline Point group & $m \overline{3} m$ & $m \overline{3} m$ & $4 / m$ & $4 / m m m$ \\
\hline Reference & $\begin{array}{c}\text { (Every \& } \\
\text { McCurdy, 1992) }\end{array}$ & $($ Nye, 1957) & $\begin{array}{c}\text { (Every \& } \\
\text { McCurdy, 1992) }\end{array}$ & $\begin{array}{c}\text { (Every \& } \\
\text { McCurdy, 1992) }\end{array}$ \\
\hline$s_{11}$ & 15.0 & 2.57 & 9.90 & 148.8 \\
$s_{22}$ & 15.0 & 2.57 & 9.90 & 148.8 \\
$s_{33}$ & 15.0 & 2.57 & 9.48 & 196.2 \\
$s_{12}$ & -6.3 & -0.73 & -4.2 & -46.0 \\
$s_{13}$ & -6.3 & -0.73 & -2.1 & -94.5 \\
$s_{23}$ & -6.3 & -0.73 & -2.1 & -94.5 \\
$s_{16}$ & 0 & 0 & 4.2 & 0 \\
$s_{44}$ & 13.3 & 6.60 & 27.1 & 153.7 \\
$s_{55}$ & 13.3 & 6.60 & 27.1 & 153.7 \\
$s_{66}$ & 13.3 & 6.60 & 24.4 & 83.2 \\
\hline
\end{tabular}


Table 2 Compliance coefficients (in $(\mathrm{TPa})^{-1}$ ) of zinc, forsterite, $\alpha$-quartz and ilmenite representing hexagonal, orthorhombic and trigonal single crystals

\begin{tabular}{ccccc}
\hline Crystal & Zinc & Forsterite* & $\alpha$-quartz & $\begin{array}{c}\text { MgSiO } \\
\text { (Ilmenite phase) }\end{array}$ \\
\hline Point group & 6/mmm & mmm & 32 & $\overline{3}$ \\
\hline Reference & $\begin{array}{c}\text { (Every \& } \\
\text { McCurdy, 1992) }\end{array}$ & $($ Bass, 1995) & $\begin{array}{c}\text { (Every \& } \\
\text { McCurdy, 1992) }\end{array}$ & $\begin{array}{c}\text { (Every \& } \\
\text { McCurdy, 1992) }\end{array}$ \\
\hline$s_{11}$ & 8.22 & 3.39005 & 12.8 & 2.6 \\
$s_{22}$ & 8.22 & 5.88334 & 12.8 & 2.6 \\
$s_{33}$ & 27.7 & 4.94942 & 9.73 & 2.7 \\
$s_{12}$ & 0.60 & -0.90936 & -1.75 & -0.97 \\
$s_{13}$ & -7.0 & -0.71289 & -1.3 & -0.30 \\
$s_{23}$ & -7.0 & -1.56059 & -1.3 & -0.30 \\
$s_{14}$ & 0 & 0 & -4.47 & 0.92 \\
$s_{15}$ & 0 & 0 & 0 & -0.82 \\
$s_{44}$ & 25.3 & 14.99250 & 20 & 10.3 \\
$s_{55}$ & 25.3 & 12.30012 & 20 & 10.3 \\
$s_{66}$ & 15.24 & 12.36094 & 29.1 & 7.14 \\
\hline$s_{i j} v$ & 0 & 0.3 &
\end{tabular}

*The $s_{i j}$ values have been obtained by inverting the $c_{i j}$ matrix reported in literature. 
Table 3 Compliance coefficients (in $(\mathrm{TPa})^{-1}$ ) of single crystals of bismuth vanadate (monoclinic) and copper sulphate pentahydrate (triclinic)

\begin{tabular}{|c|c|c|}
\hline Crystal & Bismuth vanadate & Copper sulphate pentahydrate* \\
\hline Point group & $2 / m$ & $\overline{1}$ \\
\hline Reference & (Every \& McCurdy, 1992) & (Krishnan, Radha, \& Gopal, 1971) \\
\hline$s_{11}$ & 14.7 & 29.0562 \\
\hline$s_{12}$ & 0.22 & -10.8889 \\
\hline$s_{13}$ & -6.2 & -9.24052 \\
\hline$s_{14}$ & 0 & 2.42214 \\
\hline$s_{15}$ & 6.9 & 0.682399 \\
\hline$s_{16}$ & 0 & 11.9378 \\
\hline$s_{22}$ & 10.8 & 51.9234 \\
\hline$s_{23}$ & -3.7 & -26.5755 \\
\hline$s_{24}$ & 0 & -6.6854 \\
\hline$s_{25}$ & 1.5 & -0.160799 \\
\hline$s_{26}$ & 0 & -18.35 \\
\hline$s_{33}$ & 10.0 & 39.9738 \\
\hline$s_{34}$ & 0 & 7.27663 \\
\hline$s_{35}$ & -4.8 & 3.34524 \\
\hline$s_{36}$ & 0 & 8.45123 \\
\hline$S_{44}$ & 25.5 & 60.3734 \\
\hline$s_{45}$ & 0 & -3.56223 \\
\hline$s_{46}$ & -1.0 & 0.611608 \\
\hline$s_{55}$ & 20.4 & 88.6179 \\
\hline$s_{56}$ & 0 & 23.9408 \\
\hline$s_{66}$ & 27.5 & 115.68 \\
\hline
\end{tabular}

*The $s_{i j}$ values have been obtained by inverting the $c_{i j}$ matrix reported in literature. 
Table 4 Loading directions for which Poisson's ratio is isotropic in the transverse plane for materials with cubic symmetry

\begin{tabular}{ccccc}
\hline \multirow{2}{*}{\begin{tabular}{c} 
S1. No \\
\cline { 2 - 3 }
\end{tabular}} & \multicolumn{2}{c}{ Isotropic loading directions } & & \\
\hline & $\begin{array}{c}\text { Miller indices } \\
<u v w>\end{array}$ & $\begin{array}{c}\text { Direction cosines } \\
<n_{1} n_{2} n_{3}>_{V}\end{array}$ & & $v_{W}$ \\
2 & $<100>$ & $<100>$ & 0.42 & 0.284 \\
$3 *$ & $<\frac{1}{\sqrt{3}} \frac{1}{\sqrt{3}} \frac{1}{\sqrt{3}}>$ & 0.271 & 0.284 \\
\hline$n_{1} n_{2} n_{3}>$ & $<n_{1} n_{2} n_{3}>$ & - & 0.284 \\
\hline
\end{tabular}

*A general direction of type 3 (row 3 ) is present only in crystals with $2\left(s_{11}-s_{12}\right)=s_{44}$.

Table 5 Loading directions for which Poisson's ratio is isotropic in the transverse plane for calcium molybdate $(4 / m)$

\begin{tabular}{c|ccc}
\hline \multirow{2}{*}{$\begin{array}{r}\text { Sl. } \\
\text { No. }\end{array}$} & $\begin{array}{c}\text { Miller indices } \\
<u v w>\end{array}$ & \multirow{2}{*}{ Isotropic loading directions } \\
\cline { 2 - 3 } & $<001>$ & $\begin{array}{c}\text { Direction cosines } \\
\left.<n_{1} n_{2} n_{3}\right\rangle_{V}\end{array}$ & \\
\hline 1 & $<1,-0.276143,0>$ & $<001>$ & 0.222 \\
3 & $<1,-0.72483,0>$ & $<0.80968,-0.58688,0>$ & 0.269 \\
\hline
\end{tabular}

The directions are obtained in the form of their direction cosines. The conversion to Miller indices has been done using the lattice parameters of calcium molybdate (Bass, 1995): $a=5.23 \AA$ and $c=11.44 \AA$.

Commas and minus signs (-) have been used in the Miller indices, where necessary, for clarity in this and the subsequent tables.

Table 6 Loading directions for which Poisson's ratio is isotropic in the transverse plane for indium (Point group: 4/mmm)

\begin{tabular}{|c|c|c|c|}
\hline \multirow{3}{*}{$\begin{array}{l}\text { Sl. } \\
\text { No. }\end{array}$} & \multicolumn{2}{|c|}{ Isotropic loading directions } & \multirow{3}{*}{$v$} \\
\hline & Miller indices & Direction cosines & \\
\hline & $<u v w>$ & $<n_{1} n_{2} n_{3}>_{V}$ & \\
\hline 1 & $<001>$ & $<001>$ & 0.482 \\
\hline 2 & $<1,0,0.355521>$ & $<0.93406,0,0.35711>$ & 0.464 \\
\hline 3 & $<0.908562,0.908562,1>$ & $<0.54225,0.54225,0.64181>$ & 0.421 \\
\hline
\end{tabular}

Lattice parameters of indium (Ridley, 1965): $a=4.5994 \AA$ and $c=4.9461 \AA$. 
Table 7 Definition of the parameters $a, b$ and $c$ used to simplify the solutions for orthorhombic materials

\begin{tabular}{cccc}
\hline Sl. No. & $a$ & $b$ & $c$ \\
\hline 1 & $s_{11}-2 s_{12}+s_{22}-s_{66}$ & $2\left(s_{13}-s_{23}\right)$ & $-s_{11}+2\left(s_{23}-s_{12}+s_{13}\right)-s_{22}+s_{66}$ \\
2 & $s_{11}-2 s_{13}+s_{33}-s_{55}$ & $2\left(s_{23}-s_{12}\right)$ & $-s_{11}+2\left(s_{12}+s_{23}-s_{13}\right)-s_{33}+s_{55}$ \\
3 & $s_{22}-2 s_{23}+s_{33}-s_{44}$ & $2\left(s_{13}-s_{12}\right)$ & $-s_{22}+2\left(s_{12}+s_{13}-s_{23}\right)-s_{33}+s_{44}$ \\
\hline
\end{tabular}

Table 8 Loading directions for which Poisson's ratio is isotropic in the transverse plane for forsterite (Point group $\mathrm{mmm}$ )

\begin{tabular}{cccc}
\hline \multirow{2}{*}{$\begin{array}{c}\text { Sl. } \\
\text { No. }\end{array}$} & $\begin{array}{c}\text { Miller indices } \\
\langle u v w\rangle\end{array}$ & \multirow{2}{c}{ Isotropic loading directions } \\
\hline 1 & $<0.989658,0,1>$ & $\begin{array}{c}\text { Direction cosines } \\
\left.<n_{1} n_{2} n_{3}\right\rangle_{V}\end{array}$ \\
2 & $<1,0,0.3012>$ & $<0.61839,0,0.78587>$ & 0.265 \\
3 & $<1,0.563074,0>$ & $<0.93515,0,0.35425>$ & 0.256 \\
4 & $<1,0.311763,0.392892>$ & $<0.769026,0.513977,0.3800035>$ & 0.235 \\
\hline
\end{tabular}

Lattice parameters of forsterite (Bass, 1995): $a=4.7534 \AA, b=10.1902 \AA$ and $c=5.9783 \AA$.

Table 9 Loading directions for which Poisson's ratio is isotropic in the transverse plane for the ilmenite phase of magnesium silicate $\left(\mathrm{MgSiO}_{3}\right.$, point group $\left.\overline{3}\right)$

\begin{tabular}{cccc}
\hline \multirow{2}{*}{$\begin{array}{r}\text { Sl. } \\
\text { No. }\end{array}$} & \multicolumn{2}{c}{ Isotropic loading directions } & \\
\cline { 2 - 3 } & $\begin{array}{c}\text { Miller indices } \\
<u v w>\end{array}$ & $\begin{array}{c}\text { Direction cosines } \\
\left.<n_{1} n_{2} n_{3}\right\rangle_{V}\end{array}$ & $v$ \\
\hline 1 & $<001>$ & $<001>$ & 0.111 \\
2 & $<-1,0.39982,0.595815>$ & $<-0.566978,0.163611,0.807320>$ & 0.275 \\
3 & $<1,-0.399821,0.165063>$ & $<0.898432,-0.259258,0.354408>$ & 0.200 \\
4 & $<-1,0.399819,0.228315>$ & $<-0.850951,0.245556,0.464309>$ & 0.320 \\
\hline
\end{tabular}

Lattice parameters of the ilmenite phase of $\mathrm{MgSiO}_{3}$ (Bass, 1995): $a=4.7284 \AA$ and $c=13.5591 \AA$. 
Table 10 Loading directions for which Poisson's ratio is isotropic in the transverse plane for $\alpha$-quartz (Point group 32)

\begin{tabular}{cccc}
\hline \multirow{2}{*}{$\begin{array}{c}\text { S1. } \\
\text { No. }\end{array}$} & \multicolumn{2}{c}{ Isotropic loading directions } & \\
\cline { 2 - 3 } & $\begin{array}{c}\text { Miller indices } \\
<u v w>\end{array}$ & $\begin{array}{c}\text { Direction cosines } \\
\left.<n_{1} n_{2} n_{3}\right\rangle_{V}\end{array}$ & \\
\hline 1 & $<001>$ & $<001>$ & 0.134 \\
2 & $<1,0.5,-0.484413>$ & $<-0.702479,-0.405576,0.584835>$ & -0.067 \\
\hline
\end{tabular}

Lattice parameters of $\alpha$-quartz (Bass, 1995): $a=4.1934 \AA$ and $c=5.4052 \AA$.

Table 11 Loading directions for which Poisson's ratio is isotropic in the transverse plane for bismuth vanadate (Point group 2/m)

\begin{tabular}{cccc}
\hline \multirow{2}{*}{ Sl. No. } & \multicolumn{2}{c}{ Isotropic loading directions } & \\
\cline { 2 - 3 } & $\begin{array}{c}\text { Miller indices } \\
\langle u \nu w>\end{array}$ & $\begin{array}{c}\text { Direction cosines } \\
\left.<n_{1} n_{2} n_{3}\right\rangle_{V}\end{array}$ & $v$ \\
\hline 1 & $<0.218293,0,1>$ & $<0.21772,0,0.97601>$ & 0.417 \\
2 & $<-0.885575,0,1>$ & $<-0.66819,0,0.74399>$ & 0.357 \\
3 & $<0.843115,0,1>$ & $<0.65434,0,0.7562>$ & 0.157 \\
4 & $<-1,0,0.447334>$ & $<-0.91357,0,0.40669>$ & 0.124 \\
5 & $<-0.1928,0.532064,1>$ & $<-0.12352,0.76776,0.62872>$ & 0.291 \\
\hline
\end{tabular}

Lattice parameters of bismuth vanadate (Sleight, Chen, Ferretti, \& Cox, 1979): $a=5.1935 \AA, b$ $=11.6972 \AA, c=5.0898 \AA$ and $\beta=90^{\circ} 23^{\prime} 13^{\prime}$ '.

Table 12 Loading directions for which Poisson's ratio is isotropic in the transverse plane for copper sulphate pentahydrate (Point group $\overline{1}$ )

\begin{tabular}{|c|c|c|c|}
\hline \multirow{3}{*}{ Sl. No. } & \multicolumn{2}{|c|}{ Isotropic loading directions } & \multirow{3}{*}{$v$} \\
\hline & Miller indices & Direction cosines & \\
\hline & $<u v w>$ & $<n_{1} n_{2} n_{3}>_{V}$ & \\
\hline 1 & $<0.218529,0.46844,1>$ & $<0.042341,0.61501,0.787382>$ & 0.317 \\
\hline 2 & $<0.0992786,1,0.495223>$ & $<-0.126077,0.919541,0.372222>$ & 0.333 \\
\hline 3 & $<-0.814476,-0.714811,1>$ & $<-0.31984,-0.716731,0.619677>$ & 0.325 \\
\hline 4 & $<0.39756,-0.838732,1>$ & $<0.38437,-0.838089,0.387125>$ & 0.380 \\
\hline 5 & $<1,-0.0345217,0.477128>$ & $<0.985143,-0.059928,0.160943>$ & 0.333 \\
\hline 6 & $<-1,0.103614,0.224419>$ & $<-0.865897,0.154471,0.475775>$ & 0.400 \\
\hline
\end{tabular}

Lattice parameters of copper sulphate pentahydrate (Beevers \& Lipson, 1934): $a=6.12 \AA, b=10.7 \AA$, $c=5.97 \AA, \alpha=82^{\circ} 16^{\prime}, \beta=107^{\circ} 26^{\prime}$ and $\gamma=102^{\circ} 40^{\prime}$. 


\section{REFERENCES}

Adachi, K., Ogi, H., Takeuchi, N., Nakamura, N., Watanabe, H., Ito, T., Ozaki, Y., 2018. Unusual elasticity of monoclinic $\beta-\mathrm{Ga}_{2} \mathrm{O}_{3}$. J. Appl. Phys., 124, 085102.

Ballato, A., 1996. Poisson's ratio for tetragonal, hexagonal, and cubic crystals. IEEE Transactions on Ultrasonics, Ferroelectrics, and Frequency Control 43, 56-62.

Bass, J.D., 1995. Elasticity of minerals, glasses, and melts, in: Ahrens, T.J. (Ed.), Mineral Physics \& Crystallography: A Handbook of Physical Constants, American Geophysical Union, Washington D.C., pp. 45-63.

Baughman, R.H., Shacklette, J.M., Zakhidov, A.A., Stafström, S., 1998. Negative Poisson's ratios as a common feature of cubic metals. Nature 392, 362-365.

Beevers, C.A., Lipson, H. 1934. The crystal structure of copper sulphate pentahydrate, $\mathrm{CuSO}_{4} .5 \mathrm{H}_{2} \mathrm{O}$. Proc. Roy. Soc. Lond. A 146, 570-582.

Brańka, A.C., Heyes, D.M., Wojciechowski, K.W., 2009. Auxeticity of cubic materials. Phys. Stat. Sol. B 246, 2063-2071.

Brańka, A.C., Heyes, D.M., Wojciechowski, K.W., 2011. Auxeticity of cubic materials under pressure. Phys. Stat. Sol. B 248, 96-104.

Eaton, J.W., Bateman, D., Hauberg, S., Wehbring, R., 2016. GNU Octave version 4.2.0 manual: a high-level interactive language for numerical computations. http://www.gnu.org/software/octave/doc/interpreter/.

Evans, K. E., 1991. Auxetic polymers: a new range of materials. Endeavour 15, 170-174.

Every, A.G., McCurdy, A.K., 1992. Second and higher order elastic constants, in Nelson, D.F. (Ed.), Landolt-Börnstein Tables Vol.III/29a, Springer-Verlag, Berlin, pp. 1-743.

Gunton, D.J., Saunders, G.A., 1972. The Young's modulus and Poisson's ratio of arsenic, antimony and bismuth. J. Mater. Sci. 7, 1061-1068.

Gunton, D.J., Saunders, G.A., 1975. Stability limits on the Poisson's ratio: application to a martensitic transformation. Proc. Roy. Soc. Lond. A 343, 63-83.

Hayes, M., Shuvalov, A., 1998. On the extreme values of Young's modulus, the shear modulus, and Poisson's ratio for cubic materials. J. Appl. Mech. 65, 786-787.

Krishnan, R. S., Radha, V., Gopal, E.S.R., 1971. Elastic constants of triclinic copper sulphate pentahydrate crystals. J. Phys. D: Appl. Phys. 4, 171-173.

Lethbridge, Z.A.D., Walton, R.I., Marmier, A.S.H., Smith, C.W., Evans, K.E., 2010. Elastic anisotropy and extreme Poisson's ratios in single crystals. Acta Mater. 58, 64446451.

Li, Y., 1976. The anisotropic behavior of Poisson's ratio, Young's modulus, and shear modulus in hexagonal materials. Phys. Stat. Sol. A 38, 171-175.

Love, A.E.H., 1944. A Treatise on the Mathematical Theory of Elasticity. Dover Publications, Mineola, N.Y. 
Lubarda, V.A., Meyers, M.A., 1999. On the negative Poisson ratio in monocrystalline zinc. Scr. Mater. 40, 975-977.

Marmier, A., Lethbridge, Z.A.D., Walton, R.I., Smith, C.W., Parker, S.C., Evans, K.E., 2010. ElAM: A computer program for the analysis and representation of anisotropic elastic properties. Comput. Phys. Commun. 181, 2102-2115.

Neelakantan, S., Tan, J., Markaki, A., 2015. Out-of-plane auxeticity in sintered fibre network mats. Scr. Mater. 106, 30-33.

Norris, A.N. 2006a. Extreme values of Poisson's Ratio and other engineering moduli in anisotropic materials. J. Mech. Mater. Str. 1, 793-812.

Norris, A.N., 2006b. Poisson's ratio in cubic materials. Proc. Roy. Soc. Lond. A, 462, 33853405 .

Nye, J.F., 1957. Physical Properties of Crystals: Their Representation by Tensors and Matrices. Clarendon Press, Oxford, U.K.

Paszkiewicz, T., Wolski, S., 2007a. Anisotropic properties of mechanical characteristics and auxeticity of cubic crystalline media. Phys. Stat. Sol. B 244, 966-977.

Paszkiewicz, T., Wolski, S., 2007b. Young's and shear moduli and Poisson's ratio for elastic media of high and middle symmetry. Phys. Stat. Sol. B 244, 978-987.

Poisson, S.D. 1827. Note sur l'extension des fils et des plaques élastiques. Ann. Chim. Phys. $36,384-387$.

Ridley, J., 1965. Densities of some indium solid solutions. J. Less-Common Metals 8, 354357.

Sleight, A.W., Chen, H.-Y., Ferretti, A. and Cox, D.E., 1979. Crystal growth and structure of BiVO $_{4}$. Mater. Res. Bull. 14, 1571-1581.

Ting, T.C.T., Chen, T., 2005. Poisson's ratio for anisotropic elastic materials can have no bounds. Quart. J. Mech. Appl. Math. 58, 73-82.

Turley, J., Sines, G., 1971. The anisotropy of Young's modulus, shear modulus and Poisson's ratio in cubic materials. J. Phys. D: Applied Physics 4, 264-271.

Weisstein, E.W., 2019. Quartic Equation. From MathWorld--A Wolfram Web Resource. Wolfram. 12 November. http://mathworld.wolfram.com/QuarticEquation.html (accessed 26 May 2019). 